Article

\title{
Age Estimation-Based Soft Biometrics Considering Optical Blurring Based on Symmetrical Sub-Blocks for MLBP
}

\section{Dat Tien Nguyen, So Ra Cho and Kang Ryoung Park*}

Division of Electronics and Electrical Engineering, Dongguk University, 30, Pildong-ro 1-gil, Jung-gu, Seoul 100-715, Korea; E-Mails: nguyentiendat@dongguk.edu (D.T.N.); soracho@dongguk.edu (S.R.C.)

* Author to whom correspondence should be addressed; E-Mail: parkgr@dongguk.edu; Tel.: +82-10-3111-7022; Fax: +82-2-2277-8735.

Academic Editor: Sergei Odintsov

Received: 17 June 2015 / Accepted: 10 October 2015 / Published: 19 October 2015

\begin{abstract}
Because of its many useful applications, human age estimation has been considered in many previous studies as a soft biometrics. However, most existing methods of age estimation require a clear and focused facial image as input in order to obtain a trustworthy estimation result; otherwise, the methods might produce increased estimation error when an image of poor quality is used as input. Image blurring is one of major factors that affect estimation accuracies because it can cause a face to appear younger (i.e., reduce the age feature in the face region). Therefore, we propose a new human age estimation method that is robust even with an image that has the optical blurring effect by using symmetrical focus mask and sub-blocks for multi-level local binary pattern (MLBP). Experiment results show that the proposed method can enhance age estimation accuracy compared with the conventional system, which does not consider the effects of blurring.
\end{abstract}

Keywords: human age estimation; soft biometrics; optical blurring effect; focus mask; symmetrical sub-blocks for MLBP

\section{Introduction}

With the increase of age, facial appearance changes with the manifestation of some specific features such as wrinkles, skin spots, and corners [1]. Based on this characteristic, many computer vision systems have been developed in order to accomplish several specific tasks. For example, age information is used 
in electronic consumer systems to obtain knowledge regarding customer habits, preferences, expectations, etc. [1,2]. Another useful application where age information is used is in security control and surveillance monitoring [1]. Using age information, children and youth can be restricted in accessing adult websites and movies, buying alcohol and tobacco from automated vending machines, etc. In biometrics, the age information can also be used for search-space-reduction in surveillance systems [3] and used as a demographic soft biometric [4]. In addition, many previous studies have used the human age as a soft biometric feature in order to improve the performance and reliability of classical biometric systems [1,2,4-7]. A useful application in which age information plays an importance role for ensuring recognition performance is face recognition $[1,2,8,9]$. Performance degradation in face recognition caused by age progress can be compensated by age estimation or synthesis. In the work by Batool et al. [6], the appearance of wrinkle feature according to human's age was used for recognition. They suggest that the change of face appearance according to human's age plays an important key for human identification using face. In addition to face recognition, the performance of other types of biometrics, such as fingerprints, and iris and hand geometry can be enhanced using age information [1,4].

Facial image-based age estimation can be accomplished by either active appearance model (AAM)-based or non-AAM-based methods. AAM-based methods use the statistical models of the face shape and appearance to model the face at different age values using a learning database [10-12]. After that, a set of parameters is extracted for an input facial image to model the age using the trained shape and appearance models. These methods show good estimation results. However, they have limitations when locating the facial feature points (called the landmark points) used to model the shape of the face. Several factors that can affect the locating process of the landmark points are head movement, complex background, and head pose. Therefore, although AAM-based method can produce good estimation results, it requires much time for fitting the landmark points, and it is not suitable for implementing in real-time systems.

An alternative to the AAM-based method is the non-AAM-based methods, which do not need to exactly locate the landmark points on the facial region [13-19]. Non-AAM-based methods extract the age features from the facial region without having to detect accurate positions of the landmark points.

In general, one important and visual feature that appears on the facial region as human age is the wrinkle feature. This feature appears longer, bigger, and denser on the face of elderly individuals than on that of younger people. Therefore, it is possible to predict human age through the appearance of this feature. In computer vision systems, the wrinkle feature causes high frequencies on some local face regions. Based on this fact, several high-pass filters, such as the Sobel filter, image difference, high-pass filter, and discrete wavelet transform (DWT) have been applied on several local wrinkle regions to extract the wrinkle feature [13]. After that, they used support vector regression (SVR) method on the extracted features to estimate human age. In order to extract both the appearance and directional information of wrinkle features, the Gabor filtering was used [14]. High-pass filters can successfully extract the high frequencies caused by the wrinkle feature and other skin details, but they have a limitation with the local region selection method. In previous studies, the local wrinkle regions were defined and localized using either the results of the AAM fitting procedure [13], or the correct detection results of the regions of face and eyes [14]. Instead of using local wrinkle regions, several previous studies used the entire face regions for age estimation using Gabor feature extraction method [17], subspace method [18], and bio-inspired features feature extraction method [19]. In addition, in [17], they 
removed the redundant information by a learning method that minimizing the nonlinear correlation and rank correlation between extracted features.

Recently, the local binary pattern method (LBP) has been used to extract skin detail features that contain the wrinkle and other features, such as age spots, edge-end, corner, etc. [14-16]. The advantage of the LBP method is that it can extract both local and global skin features by dividing the facial region into local sub-blocks. In addition, it can be applied in the facial rectangular region without the detection of accurate positions of facial feature points such as eyebrows, mouth, etc. by AAM. By applying the multi-level approach on the LBP method, estimation results are greatly enhanced. In Table 1, we summarize some results of previous studies on age estimation problem using different feature extraction methods and datasets. The smaller value of mean absolute error (MAE) indicates the better estimation performance.

Table 1. A summary of estimation performance of previous studies on age estimation problem.

\begin{tabular}{|c|c|c|c|}
\hline Studies & Dataset & Feature Representation & MAE (Years) \\
\hline Lanitis et al. [12] & $\begin{array}{l}400 \text { images from } \\
40 \text { individuals }\end{array}$ & AAM Feature & 3.82 \\
\hline \multirow{2}{*}{ Choi et al. [13] } & BERC & Gaussian High-Pass Filter Feature (GHPF) & 6.85 \\
\hline & PAL & Gaussian High-Pass Filter Feature (GHPF) & 8.44 \\
\hline Nguyen et al. [14] & PAL & MLBP and Gabor Filtering Features & 6.52 \\
\hline Li et al. [17] & FG-NET & $\begin{array}{l}\text { Gabor filter with feature selection method } \\
\text { (PLO Feature) }\end{array}$ & 4.82 \\
\hline \multirow{4}{*}{ Geng et al. [18] } & \multirow{2}{*}{ MORPH } & Aging Pattern Subspace (AGES) & 8.83 \\
\hline & & AGES (With Linear Discriminant Analysis) & 8.07 \\
\hline & \multirow{2}{*}{ FG-NET } & Aging Pattern Subspace (AGES) & 6.77 \\
\hline & & AGES (With Linear Discriminant Analysis) & 6.22 \\
\hline Guo et al. [19] & FG-NET & Bio-Inspired Features (BIF) & 4.77 \\
\hline
\end{tabular}

Although the above age estimation methods have shown good estimation results, estimation performance is affected significantly by the quality of captured face images. Several factors, such as occlusion of the face region caused by hair, head pose, or the use of a facemask, in addition to blurring effects, can produce the disappearance of age information on a captured face. Consequently, the estimation system can produce a large error when estimating age. Image blurring is one of the main factors. Because of the user's head movement and the limitation of the camera's depth-of-field (DOF), image blurring occurs frequently when capturing user facial images for age estimation. Because of blurring, the distinctiveness of the face boundary is reduced. This is a key problem of the AAM-based method to obtaining good estimation results. In addition, blurring also causes the disappearance of small skin details and makes the level of the captured wrinkle feature to be weak. Consequently, the non-AAM-based method also fails to correctly estimate the age of people in blurred images used as input. Nevertheless, to the best of our knowledge, there is no previous research for age estimation that considers image blurring.

Therefore, we propose a new human age estimation method that is robust to optical blurring effect by using focus mask and sub-blocks for multi-level local binary pattern (MLBP). The proposed method is novel when compared with previous studies in the following two ways.

- The proposed method assesses the degree of blurring of the input facial image using focus score (FS) measurement. Then, we classify the input facial image into one of several groups in terms 
of degree of blurring based on the measured FS. Furthermore, we estimate the human age using an age estimator optimized with the data from the classified group.

- By considering the overlapped area between two neighbor groups of images that have similar blurring degree, the misclassification error of the two groups is reduced. If the measured FS belongs to the overlapped area of the two groups, two age values are estimated by two age estimators optimized with each group, and these two values are combined by score level fusion, which enhances the accuracy of age estimation.

In Table 2, we summarize the previous and proposed methods on age estimation.

Table 2. Summary of previous and proposed studies on age estimation.

\begin{tabular}{|c|c|c|c|}
\hline Category & Method & Strength & Weakness \\
\hline $\begin{array}{l}\text { AAM-based } \\
\text { method }\end{array}$ & $\begin{array}{l}\text { Model the shape and } \\
\text { appearance of the } \\
\text { human face } \\
\text { using statistical } \\
\text { models [10-12] }\end{array}$ & $\begin{array}{l}\text { Produce a good } \\
\text { estimation result with } \\
\text { clear and good quality } \\
\text { input images }\end{array}$ & $\begin{array}{l}\text { Estimation accuracy is } \\
\text { affected by the detection } \\
\text { results of landmark points } \\
\text { whose performance can be } \\
\text { affected by image blurring, } \\
\text { complex background, head } \\
\text { movements, etc. } \\
\text { - Significant processing time } \\
\text { is required for model } \\
\text { fitting. } \\
\text { Estimation accuracy is } \\
\text { degraded significantly with } \\
\text { blurred image }\end{array}$ \\
\hline \multirow[t]{2}{*}{$\begin{array}{l}\text { Non-AAM-based } \\
\text { methods }\end{array}$} & $\begin{array}{l}\text { Employs single-level } \\
\text { LBP on entire face } \\
\text { region [15], } \\
\text { MLBP [16], and } \\
\text { combination of } \\
\text { MLBP and Gabor } \\
\text { filtering [14], Gabor } \\
\text { feature [17], } \\
\text { subspace } \\
\text { method [18]. }\end{array}$ & $\begin{array}{l}\text { Age estimation result is } \\
\text { not affected significantly } \\
\text { by accurate localization } \\
\text { of the face region and } \\
\text { facial features } \\
\text { - } \quad \text { Processing speed is faster } \\
\text { than AAM-based method }\end{array}$ & $\begin{array}{l}\text { Estimation accuracy is } \\
\text { affected significantly by } \\
\text { image blurring }\end{array}$ \\
\hline & Proposed method & $\begin{array}{l}\text { Employs the advantages } \\
\text { of the non-AAM-based } \\
\text { methods } \\
\text { - } \quad \text { Robust to image blurring }\end{array}$ & $\begin{array}{l}\text { Additional procedure for } \\
\text { measuring the FS for the } \\
\text { image is required }\end{array}$ \\
\hline
\end{tabular}

The remainder of this paper is organized as follows: In Section 2, the proposed system for age estimation is described. In Section 3, the experiment setup and results are discussed. Finally, the conclusions are presented in Section 4. 


\section{Proposed Method}

\subsection{Overview of the Proposed Method}

In order to manage the effects of image blurring, we propose a new human age estimation method based on image focus measurement, as depicted in Figure 1. The first step in our method is the detection and localization of the face region. This is the required step of all face image-based age estimation systems because age information appears only on the face region. Because of the natural head pose of humans when capturing the face, in-plane rotation (roll) can occur. Therefore, in addition to face detection, we implement an in-plane rotation compensation and face region redefinition in order to carefully estimate the face region before further processing steps. The explanation for these steps is provided in more detail in Section 2.2.

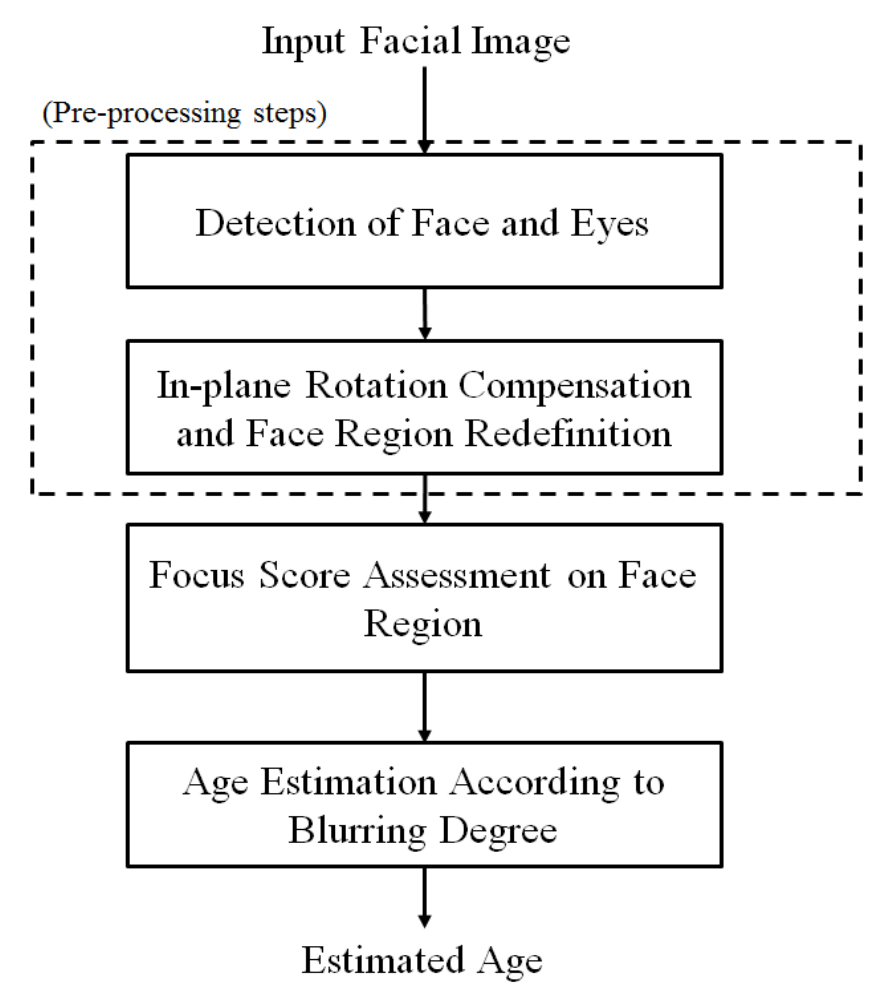

Figure 1. Overall procedure for the proposed method for human age estimation.

In the next step, our method assesses the FS of the face region that represents the focus quality of the face. As explained in Section 1, image blurring affects significantly the performance of age estimation systems because of the disappearance of age information on blurred images. Therefore, in this research, we pre-classify input faces into several groups according to blurring degree before performing the age estimation procedure based on the FS measurement. For each group, an appropriate age estimator is used to estimate the age of the input face. 
If the age estimation system is trained using only focused images, blurred images can result in an elderly individual being recognized incorrectly as a young person because the wrinkle and spot information of the older person is not seen in the blurred image. Although the system can be trained using both focused and non-focused images in order to solve this problem, estimation accuracy inevitably becomes lower with a large variation in the estimated age because of a large variation of training images.

Therefore, by pre-classifying the input faces into several groups by blurring degree, we can reduce the variation of the input faces. In addition, although the face images are blurred, the images at different age steps that have a similar blurring degree can contain sufficient information for age discrimination. As a result, each group with face images of similar blurring degree can be modeled through an appropriate age estimator. The details of the focus measurement are provided in Section 2.3, and those for the age estimation method are provided in Section 2.4.

\subsection{Preprocessing Steps for Face Detection and Face Region Redefinition}

Normally, the input face image not only contains the face region, but also the background area. Therefore, the first step of face image-based age estimation systems is the detection and localization of the face region [14]. In our method, we use the adaptive boosting (Adaboost) method for this step [20]. The Adaboost method is based on Haar-like features to construct simple face classifiers. Then, a strong face classifier is built by cascading several simple classifiers. The Adaboost method is used widely because it can produce good detection results, and it is suitable for real-time processing. Based on the detected face region, we also locate both eyes using the Adaboost eye classifier within the predefined area inside the detected face region. We use the face and eye Adaboost detectors provided by the OpenCV library (ver. 2.4.9) [21]. Then, the compensation of in-plane rotation of the face area and redefinition of the face region are performed as follows [14]. In-plane rotation can usually be presented on the face region because of the natural human head pose. This type of rotation results in the misalignment of faces, and thus, it reduces estimation performance [22]. Therefore, our method compensates for in-plane rotation using the detection results of two eyes, as shown by Equation (1).

Suppose that we detect the left and right eyes using the Adaboost method. In-plane rotation compensation is performed by rotating the face region by an angle $\theta$, calculated by Equation (1). In this equation, $\left(R_{x}, R_{y}\right)$ and $\left(L_{x}, L_{y}\right)$ indicate the coordinates of the detected left and right eyes, respectively, and $\theta$ is the rotation angle.

$$
\theta=\tan ^{-1}\left(\frac{R_{y}-L_{y}}{R_{x}-L_{x}}\right)
$$

As the final step of face localization, we redefine the ROI of face region based on face geometry using the position of the two eyes, as shown in Figure 2. The step for redefining the face ROI is required because the box detected by the Adaboost face detector normally does not fit to the actual position of the face. In Figure 2, $d$ indicates the measured distance between the two eyes. Based on this value, we experimentally define the ratio values $k_{1}, k_{2}$, and $k_{3}$ to define the geometry of the face region. Therefore, these values are person-independent. In our experiments, we set $k_{1}, k_{2}$ and $k_{3}$ ratios to $0.5,0.75$ and 1.5 , respectively. 


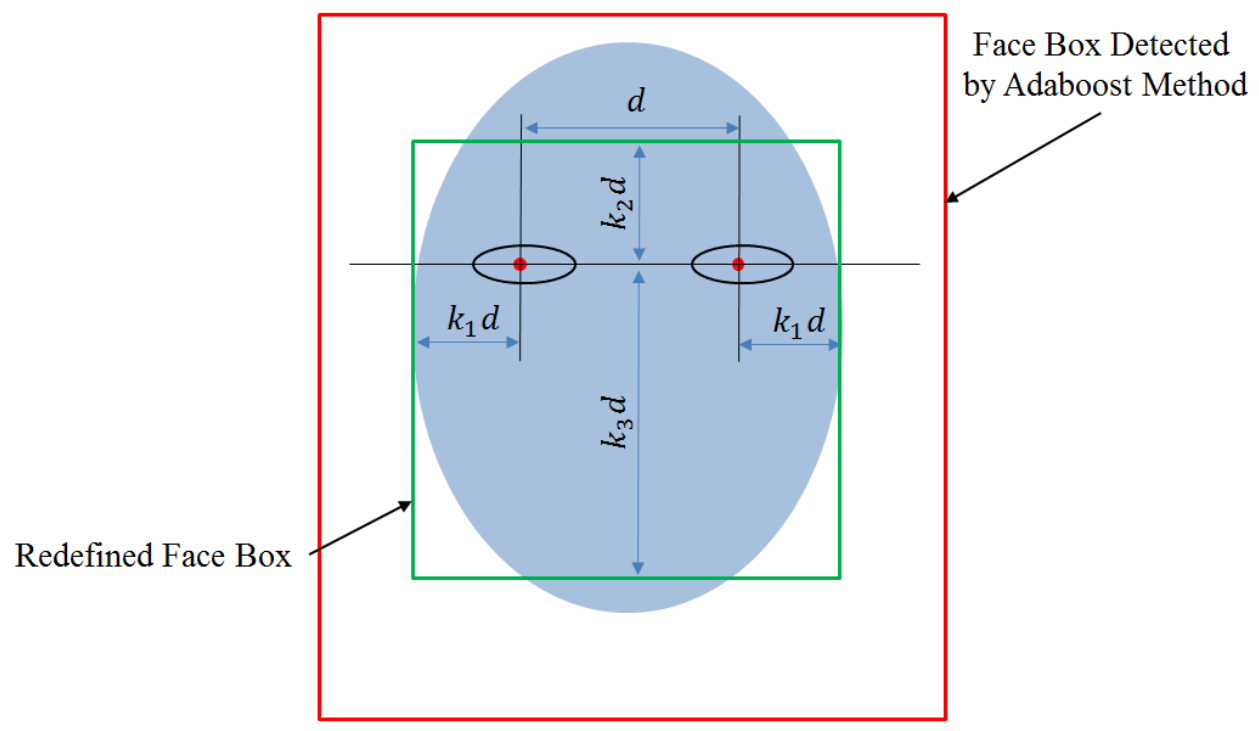

Figure 2. Methodology of redefining face region-of-interest (ROI) based on the detection results of the eyes and face.

\subsection{Estimation of the Degree of Image Blurring by FS Measurement}

An observed image $(g(x, y))$ is usually modeled by a convolution of original scene $(f(x, y))$ with a point-spread function (PSF) of blurring $(h(x, y))$, as shown in Equation (2). The PSF function has the characteristic of low-pass filters [23] that describe the response of the capturing system to a point in the original scene. However, in actual cases, the PSF function causes the spread of original points, and the observed image becomes blurred.

$$
g(x, y)=f(x, y) \times h(x, y)
$$

Based on Equation (2), the estimation method for the blurring degree focuses on measuring the amount of high-frequency components on the observed image. In order to measure the amount of high-frequency components, various methods for measuring FS based on the sum-modified-Laplacian (SML) [24], wavelet transform [25], and convolution mask [26,27] were proposed. In our method, we use the FS measurement proposed by Kang et al. [26] because of the advantages of fast processing speed and high performance of focus assessment. Kang's method is used to assess the FS of iris images by applying the convolution mask shown in Figure 3 on the iris region. Different from this, in our method, we use Kang's method to assess the FS of face ROI, which is obtained as shown in Section 2.2.

\begin{tabular}{|l|l|l|l|l|}
\hline-1 & -1 & -1 & -1 & -1 \\
\hline-1 & -1 & +4 & -1 & -1 \\
\hline-1 & +4 & +4 & +4 & -1 \\
\hline-1 & -1 & +4 & -1 & -1 \\
\hline-1 & -1 & -1 & -1 & -1 \\
\hline
\end{tabular}

Figure 3. Convolution mask for assessing focus score (FS). 
By the convolution operation with the mask shown in Figure 3 in the face ROI of Figure 2, we measure the amount of high-frequency components included in the face ROI, and determine the total amount of high-frequency components as a total power value. Finally, FS is calculated using a nonlinear normalized function proposed by Daugman [27], as shown in Equation (3).

$$
\mathrm{FS}=100 \times\left(\frac{x^{2}}{x^{2}+c^{2}}\right)
$$

In Equation (3), $c$ is the normalization offset that determines the slope of the non-linear normalization function, and the optimal $c$ value is determined experimentally. In order to find the best value for $c$ in our method, we performed various experiments with different $c$ values; then, we selected the optimal offset $c$, through which we achieved the best classification performance of blurring images. $x$ is the total power value measured by the mask for Kang's method in the face ROI. In previous studies [26,27], they measured the focus score of iris image, but we applied the method on the face image. Most part of iris image usually includes the mid or high frequency components caused by iris textures, eyelashes, and eyelids. However, the face image includes the large part of left and right cheeks which produce the low frequency component due to less amount of texture than eyes, nostrils and mouth. Therefore, these parts of cheeks cannot provide the accurate focus score by using the mask of Figure 3.

In order to solve this problem, we assign the larger weights to the mask values, which are calculated in the areas of eyes, roughly defined regions of nostrils and mouths (having larger amount of high frequency components), whereas the smaller weights are assigned to the mask values calculated in the regions of cheeks. In details, based on the detection result of face and eyes, we roughly define the eyes, nose and mouth regions. On these regions, the convolution result is multiplied by the weight of 2 , whereas those of other skin regions are multiplied by the weight value of 1 . These weights were experimentally determined. This is the main difference between our focus evaluation and previous research [26,27].

In Figure 4, we show examples of FS measurement for face images according to blurring degree. As shown in the figure, the blurred images have smaller FS values compared with the focused images.

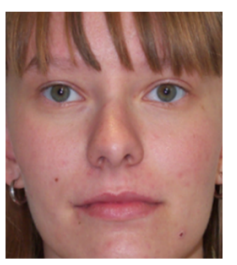

$\mathrm{FS}=99$

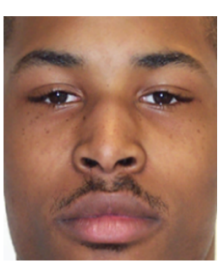

$\mathrm{FS}=99$

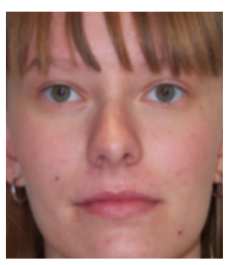

$\mathrm{FS}=87$

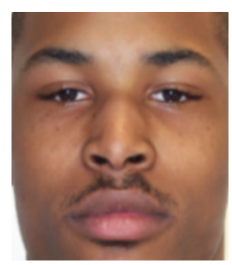

$\mathrm{FS}=93$

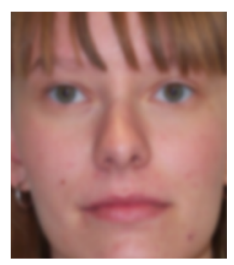

$\mathrm{FS}=23$

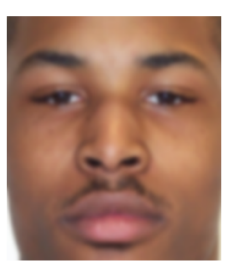

$\mathrm{FS}=38$

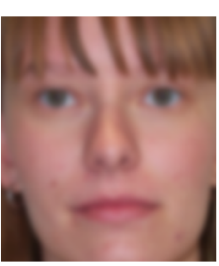

$\mathrm{FS}=4$

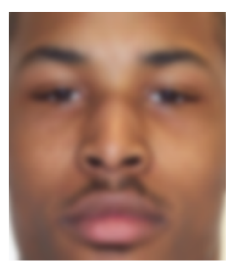

$\mathrm{FS}=7$

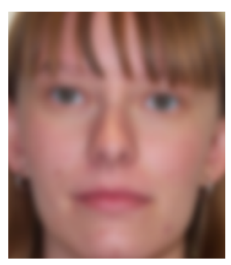

$\mathrm{FS}=1$

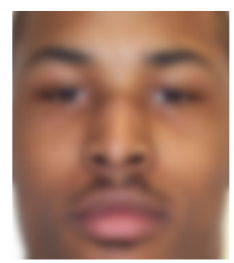

$\mathrm{FS}=2$

Figure 4. Examples of FS measurement for images at various blurring degrees using Kang's method; $c$ value is set to 1200 . 
Based on FS measurements for the face ROI, our method classifies input faces into one of several blurring degree groups. In other words, we roughly define three groups of blurring degrees, focused, slightly blurred, and blurred groups, using the training data. The focused group contains focused images, whereas the slightly blurred and blurred groups contain slightly and significantly blurred images, respectively. For example, in Figure 4, the first two images in each row belong to the focused group, the third image in each row belongs to the slightly blurred group, and the last two images in each row belong to the blurred group. Ideally, we can set hard boundaries (thresholds) between each group through which the images are correctly classified into one of the three groups. However, in actual cases, the measured FS for the face ROI can have variations, even with face images that have the same degree of blurring, according to the inclusion of hair, the use of facemasks, etc. Consequently, hard boundaries are difficult to set, and misclassification can occur.

In order to solve this problem, we propose a scheme that considers the overlapped areas between two groups, as shown in Figure 5. Instead of using hard thresholds to classify images into one of the three groups, we use several thresholds to classify the images into correct and overlapped groups.

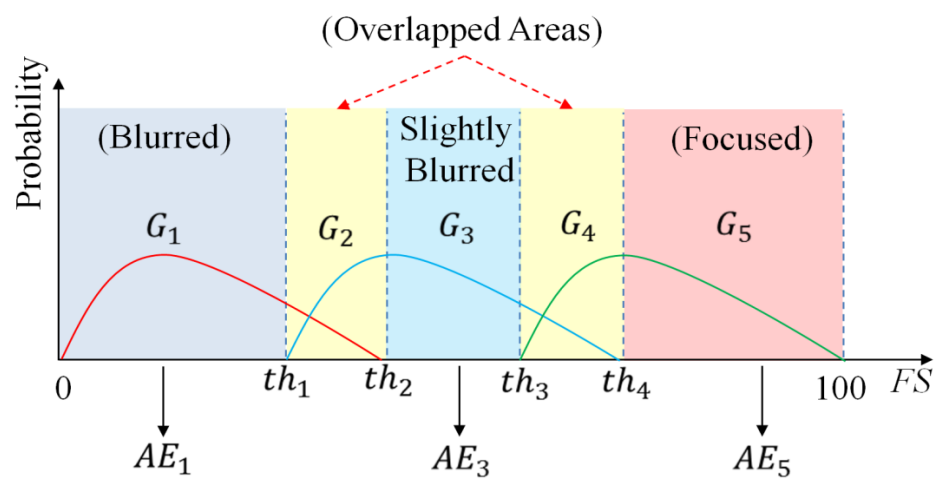

Figure 5. Description of our proposed method for age estimation through the pre-classification of images into one of five groups for blurring degree.

In Figure 5, we show an example of applying our scheme to the three groups of focused, slightly

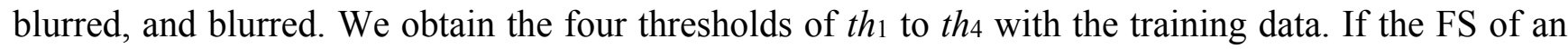
image is greater than $t_{4}$, the image is determined as belonging to the focused group $\left(G_{5}\right)$. If the FS is between $t h_{2}$ and $t h_{3}$, the image is determined as belonging to the slightly blurred group $\left(G_{3}\right)$. If the FS is smaller than $t h_{1}$, the image is determined as belonging to the blurred group $\left(G_{1}\right)$. Then, we obtain three age estimators ( $A E_{1}, A E_{3}$, and $A E_{5}$ in Figure 5) optimized with the training data of $G_{1}, G_{3}$, and $G_{5}$, respectively, and the age of an input image is estimated using the corresponding age estimator. Detailed explanations of our age estimator are provided in Section 2.4. In addition, there are two cases where the FS of an image is between $t h_{1}$ and $t h_{2}\left(G_{2}\right)$, or between $t h_{3}$ and $t h_{4}\left(G_{4}\right)$. In these cases, the images are determined as belonging to the overlapped groups of $G_{2}$ and $G_{4}$, and the age of the face image is obtained by combining the two estimated ages by the age estimators of the two neighboring groups. For example, if the FS of an image belongs to $G_{4}$, the final age of the face image is obtained by combining the two ages estimated by $A E_{3}$ and $A E_{5}$. A detailed explanation of such combination is demonstrated by Equations (13)-(15) in Section 3.3. The optimal thresholds $t h_{1}$ to $t h_{4}$ are determined with training data. 
Based on this scheme, we can classify the input face images into one of the five groups of blurring degree. By considering the overlapped groups, the FS variation of the input images can be reduced. In addition, by applying an appropriate age estimator for each group, the age estimation accuracy is enhanced compared with the methods that do not consider the effects of blurring.

\subsection{Age Estimator Based on MLBP, Gabor Filtering, PCA, and SVR}

In a previous study [28], the symmetry was established as a dynamic feature in artificial vision. They showed that the symmetry is an important feature and could be used for expression estimation using face image. In addition, they claimed that the human face is mostly appeared symmetrically. In another study [29], the symmetrical property of object's parts was used to detect the objects.

Based on these studies [28,29], we attempt to extract a strong image feature for human age estimation problem from symmetrical face. For this purpose, we first perform an in-plane rotation procedure to compensate the in-plane rotation of face and make the face region appears in symmetrical shape. Then, we redefine the face ROI to obtain the optimal face region based on the symmetrical characteristic of human face. In addition to that, we use the MLBP feature extraction method to extract the image features by dividing the face region into symmetrical sub-blocks and concatenating the extracted features of all sub-blocks together. Using that procedure, we can extract the image features that appear in local symmetrical sub-blocks. Consequently, the image features are obtained not only from global symmetrical face region, but also the local symmetrical sub-blocks on face. This method helps our method capture both global and local feature from face for age estimation problem. In the experimental result part, we show that our approach can produce the better estimation results compared to previous research. Therefore, we use the terms of symmetrical sub-blocks in our paper.

In this section, we briefly describe our age estimator based on the feature extraction by MLBP and Gabor filtering, and SVR methods [14]. The overall procedure of our age estimation method is depicted in Figure 6. As shown in the figure, the proposed method uses SVR to build an age estimation model based on the extracted age feature. In other words, the MLBP-based feature extraction method is used to obtain the global texture feature, whereas Gabor filtering is used to extract the local wrinkle feature. With the two types of extracted features, we form the final feature by combining the two. After that, we use PCA method to reduce the dimension of features. Finally, the features after PCA are used as the SVR input for estimating age.

In previous research [14], features are combined through MLBP and Gabor filtering by concatenating for age estimation, which increases the dimension of the features and consequent processing time. Therefore, in order to reduce the dimension of the extracted age feature and obtain the optimal features, we newly apply the PCA method on the concatenated features before estimating the age using SVR. Using PCA method, the dimension of the age feature is reduced, and the performance of the estimation system is enhanced. The optimal number of eigen-vectors by PCA is determined with the training data by which the smallest age estimation error is obtained. 


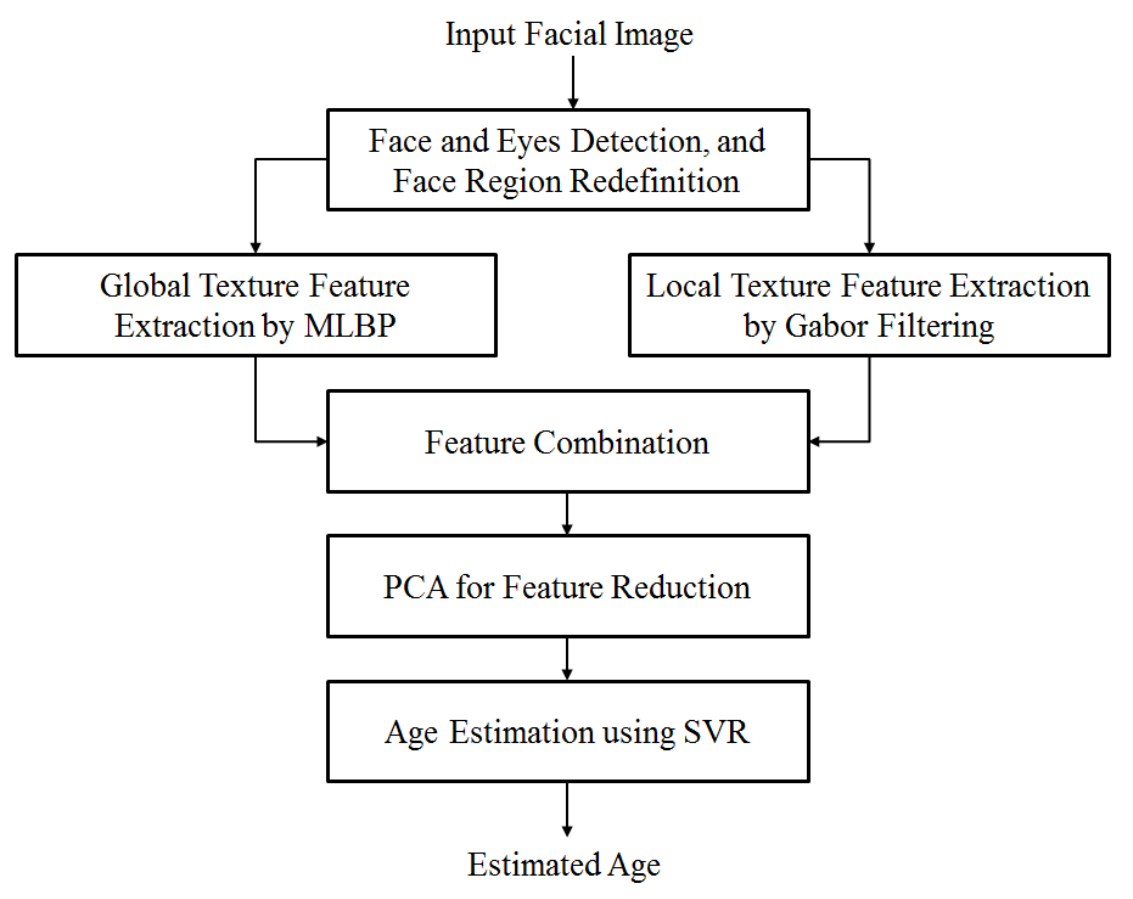

Figure 6. Overall procedure for age estimation method.

\subsubsection{Global Texture Feature Extraction by MLBP Method}

LBP is used widely to extract image textures because of its various advantages, including illumination and rotation invariants [14,30]. The LBP method is used in many applications, such as finger-vein [31], gender [32,33], facial expression [34], and face recognition [35], in addition to age estimation [14,15]. The LBP method encodes each pixel in a given image with a combination of $P$ ( $P$ bits) by comparing the $P$ surrounding pixels on a circle of radius $R$ with the center pixel. Mathematically, the LBP operator is defined as in Equation (4).

$$
L B P_{R, P}=\sum_{i=0}^{i=(P-1)} s\left(g_{i}-g_{c}\right) \times 2^{i}
$$

In Equation (4), $P$ and $R$ indicate the number of surrounding pixels and the radius of the circle from where the surrounding pixels are obtained, respectively. The $g_{c}$ and $g_{i}$ values are the gray levels of the center pixel and the $i$ th surrounding pixel, respectively. In addition, $\mathrm{s}(x)$ is a threshold function that returns the value of zero if the input is smaller than zero; otherwise, it returns the value of one.

In addition to providing illumination and rotation invariant ability, the LBP descriptor is useful for representing the microstructure of the image texture, such as the age spot region, edge, and corner $[14,15]$. This ability is more suitable for age estimation using texture information. As human's age, the facial skin changes by presenting some special features, such as wrinkles and bigger age spots. Consequently, the quantity of such features present on a face can be used to predict human age. The LBP descriptor can be classified into uniform and non-uniform patterns. The uniform patterns are the micro-textures that contain at most two bit-wise changes from zero to one or one to zero. Other patterns with a larger number of bit-wise changes are regarded as the non-uniform pattern. The reason for this classification derives from the observation that uniform patterns are useful for describing age texture features, such as wrinkles, 
corner, and spots, whereas non-uniform patterns do not have sufficient information to describe these texture features. Using the uniform and non-uniform patterns, age features are formed as a histogram of patterns [14]. In Figure 7, we show the methodology for extracting the LBP feature from a face ROI. In this figure, we divide the face ROI into $4 \times 4$ symmetrical sub-blocks, and obtain texture feature histograms from each sub-block (not from the entire face ROI). The histograms from each sub-block are concatenated as shown in Figure 7.

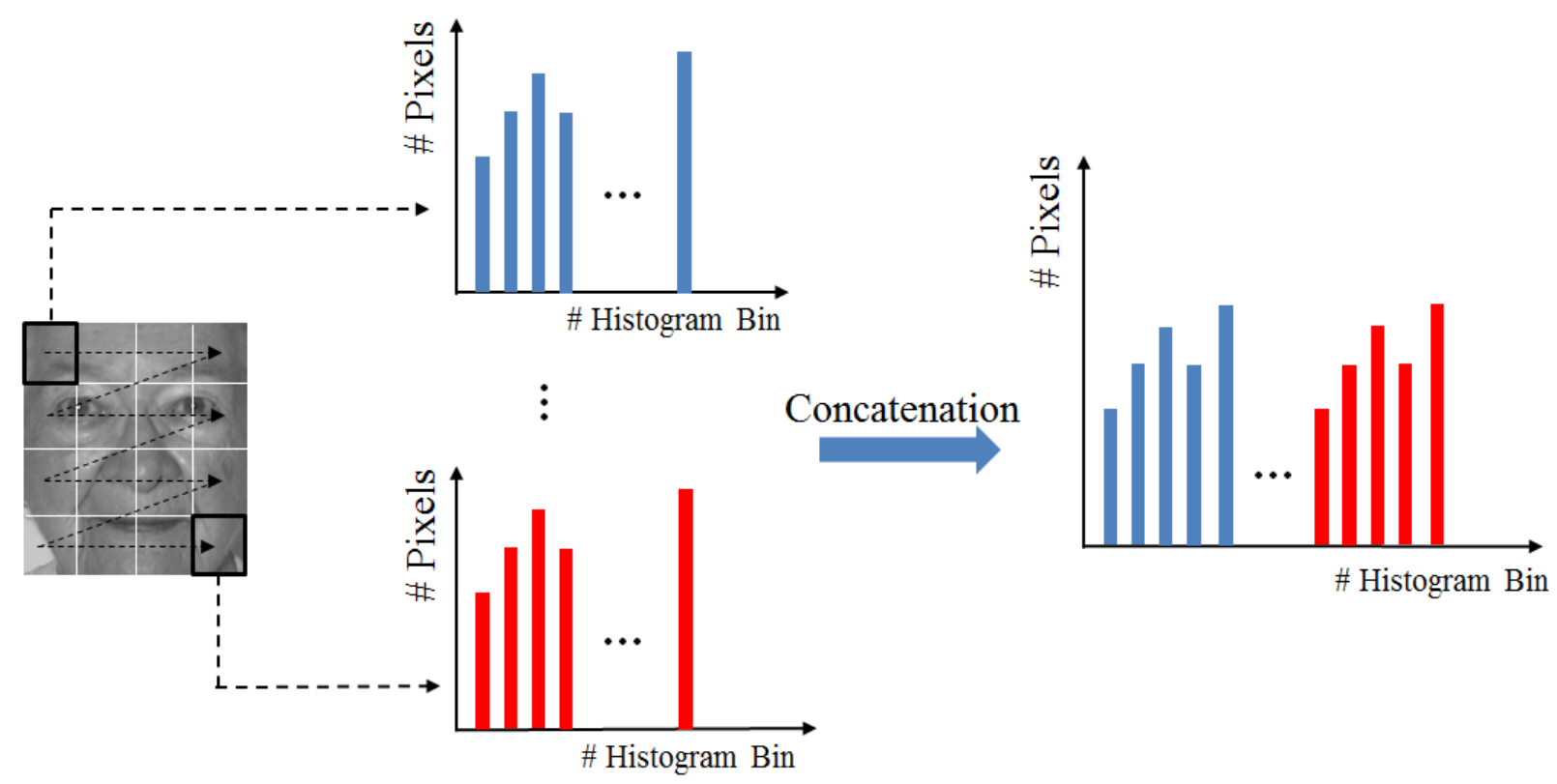

Figure 7. Local binary pattern method (LBP) feature formation from symmetrical sub-blocks for age estimation.

With the single-level LBP histograms of Figure 7, it is difficult to obtain all the age information from various scales. Therefore, we use the MLBP method [14,35]. The methodology for the MLBP feature formation is depicted in Figure 8. Intuitively, MLBP is composed of several single-level LBP features obtained with different the parameters of radius $(R)$, number of surrounding pixels $(P)$, and number of sub-blocks $(M)$. The MLBP method can capture age features at different scales $(R)$ and resolution $(P)$ of the LBP operator, and the size of the local region. By concatenating the LBP histograms from each level, as shown in Figure 7, both the global and local texture information can be extracted by the MLBP method. Consequently, richer age information can be obtained through MLBP compared with the LBP method.

\subsubsection{Local Age Texture Feature Extraction by Gabor Filtering}

The wrinkle feature is one of the most important age features that appear on a human face as human's age. This feature is weak (small and short) on young people, and becomes longer and stronger on older people. Although the MLBP feature can describe the appearance of texture features robustly to illumination and rotation variations, it has a limitation in describing the wrinkle feature. This is because the LBP descriptor is extracted by comparing the surrounding pixels with the center pixel. Consequently, a strong wrinkle area can produce an LBP descriptor that is similar to a weak wrinkle region. In Figure 9, we show an example of this problem. As shown in this figure, although the wrinkle features of the older 
person in the first row of the figure are extremely strong (long and large depth) compared with those of the younger person in the second row of the same figure, the LBP descriptors of these two wrinkle features are the same. To overcome this problem, we use Gabor filtering to extract the local wrinkle feature, in addition to the MLBP feature.
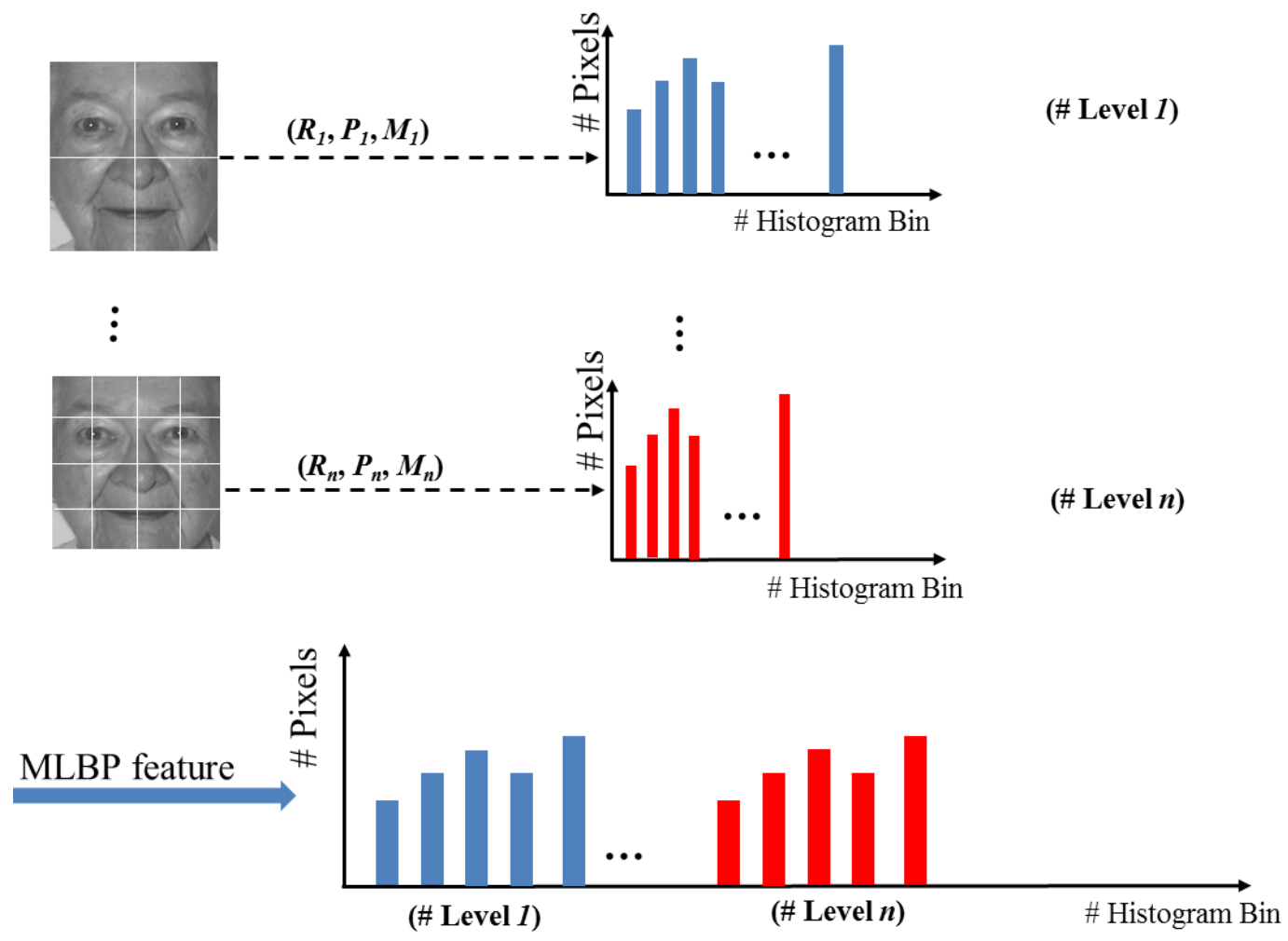

Figure 8. (Multi-level local binary pattern) MLBP feature formation from symmetrical sub-blocks for age estimation.
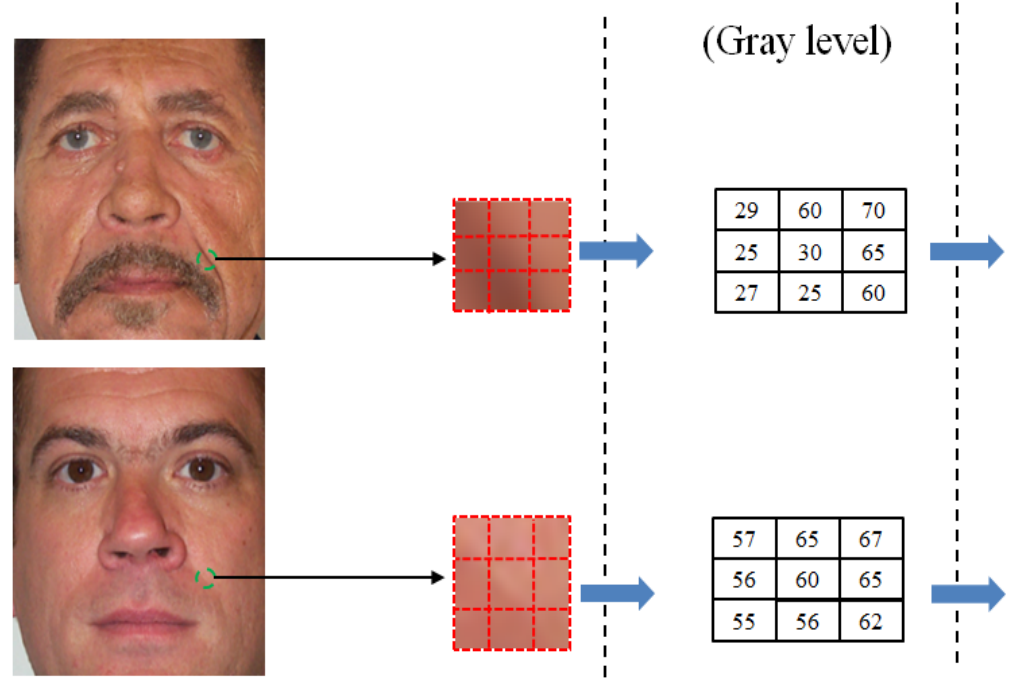

(LBP Descriptor)

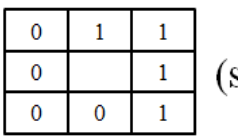
(strong edge)

\begin{tabular}{|l|l|l|}
\hline 0 & 1 & 1 \\
\hline 0 & & 1 \\
\hline 0 & 0 & 1 \\
\hline
\end{tabular}
(weak edge)

Figure 9. Example of the limitations of the LBP feature to describe the strength of the wrinkle feature. 
Equation (5) represents a two-dimensional Gabor filter composed of a Gaussian kernel multiplied by a sinusoid wave [14,36]. In this equation, $\sigma_{x}$ and $\sigma_{y}$ indicate the standard deviations of the filter in the $x$ - and $y$-axes, respectively. Furthermore, $W$ is the sinusoid frequency.

$$
G(x, y)=\frac{1}{2 \pi \sigma_{x} \sigma_{y}} \exp \left\{-\frac{1}{2}\left(\frac{x^{2}}{\sigma_{x}^{2}}+\frac{y^{2}}{\sigma_{y}^{2}}\right)+j 2 \pi W x\right\}
$$

In order to extract the wrinkle feature, we first define several local wrinkle regions based on the position of the eyes, where the wrinkle feature normally appears on a face, as shown in Figure 10. Then, the high-frequency components in the region are extracted by the convolution operation with the Gabor filter. Finally, the mean and standard deviation of the filtered image are used as the extracted wrinkle feature. For fast processing, we only use the real part of Gabor filtering, and the kernel parameters are obtained from previous research.

$$
R(x, y)=\frac{1}{2 \pi \sigma_{x} \sigma_{y}} \exp \left\{-\frac{1}{2}\left(\frac{x^{2}}{\sigma_{x}^{2}}+\frac{y^{2}}{\sigma_{y}^{2}}\right)\right\} \cos (2 \pi W x)
$$

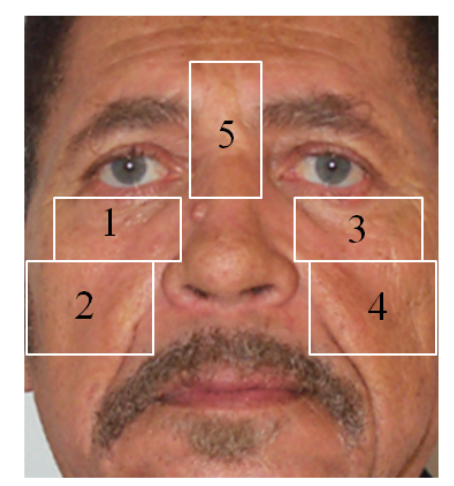

Figure 10. Definition of five selected local wrinkle regions with our method.

\subsubsection{Optimal Feature Selection by PCA and Age Estimation Using SVR}

Using MLBP and Gabor filtering, our age estimation method extracts two types of texture features. In order to estimate human age, we first normalize the two extracted features using Z-score normalization. Suppose we have a raw feature vector $f_{i}$ extracted from an image. Normalization is conducted using Equation (7). In this equation, the values of $\boldsymbol{\mu}_{i}$ and $\boldsymbol{\sigma}_{\boldsymbol{i}}$ indicate the mean and standard deviation vectors of the feature vectors [14], respectively.

$$
f_{i}^{*}=\frac{f_{i}-\mu_{i}}{\sigma_{i}}
$$

In Equation (7), $i=1$ in the case where the feature vector is extracted by MLBP, and $i=2$ when that is extracted by the Gabor filter. After normalizing the raw feature vectors, we combine the normalized feature vectors to form the final age feature vectors. The combination is done simply by concatenating two normalized vectors, as shown in Equation (8) [14]. In this equation, because we have two types of texture feature vectors by MLBP and Gabor filtering, the combination is done by concatenating these two types. 


$$
\boldsymbol{f}=\left[\boldsymbol{f}_{1}{ }^{*}, \boldsymbol{f}_{2}{ }^{*}\right]
$$

As shown in Figures 7 and 8, because we divide the face ROI into several local regions (sub-blocks) to obtain the MLBP features, the dimension of the MLBP features is increased. In details, if we extract the single-level LBP feature of a face image using P surrounding pixels of LBP operator of Equation (4). Then, the number of uniform rotation-invariant codes is $(P+1)$ and the number of non-uniform rotation-invariant code is one. Totally, we extract $(\mathrm{P}+2)$ histogram bins for the image. Consequently, for example, if we divide the face image into 100 sub-blocks and use the LBP operator with radius one and eight surrounding pixels ( $\mathrm{P}$ is set to 8 in this case), the consequent dimension of the extracted LBP feature is 1000 (100 sub-blocks $\times 10$ histogram bins). In addition, if we use three single-level LBP features to construct an MLBP feature, the dimension of the MLBP feature is much larger than 1000. As shown in Equation (8), the final texture feature vector is constructed by concatenating the MLBP and Gabor feature vectors, and the dimension of the final feature vector is extremely high. The high dimensionality of the feature vector not only requires significant processing time for SVR training and testing, but it can also degrade the estimation performance. In addition, redundant features exist among these feature vectors.

To solve this problem, we use the PCA method to reduce the feature dimension and obtain the optimal features before using SVR for age estimation. PCA is a well-known method that finds a new low-dimensional data space where the data are best represented [37-40]. The new data space is formed by a set of $n$ orthogonal base vectors, called $\boldsymbol{u}_{i}$. In order to find these base vectors, the PCA method first calculates the covariance matrix $\boldsymbol{C}$ using the training data according to Equation (9) [38].

$$
\boldsymbol{C}=\frac{1}{N} \sum_{i=1}^{N} \boldsymbol{x}_{\boldsymbol{i}} \boldsymbol{x}^{T}{ }^{T}
$$

In this equation, $T$ indicates the transpose operator, $N$ is the number of feature vectors in the training data, and $\boldsymbol{x}_{\boldsymbol{i}}$ is a feature vector obtained using Equation (8). With the covariance matrix $\boldsymbol{C}$, the $n$-base vectors of the new data space are found by solving the eigen-value and eigen-vector problems of matrix $\boldsymbol{C}$. Because $\boldsymbol{C}$ is a non-negative symmetric matrix, it has all the non-negative eigen-values $\lambda_{i}$, and the corresponding eigen-vectors $\boldsymbol{u}_{i}$. Suppose that $\lambda_{\mathrm{i}} \geq \lambda_{\mathrm{j}}$ with $i>j$. Then, the $n$-base vectors of the new data space are the corresponding eigen-vectors $\boldsymbol{u}_{i}$ of eigen-values $\lambda_{i}$. The matrix $\boldsymbol{W}$ used to transform a vector from the original data space into a vector in the new data space is given in Equation (10). For dimension-reduction purposes, we can experimentally choose a small value $n$ by which amount of lost data is acceptable. A small value of $n$ causes a large amount of data to be lost. However, an extremely large value of $n$ causes new data associated by noise. In order to find the best value for $n$, we perform various experiments with different $n$ values, and select the optimal $n$ value with which the best accuracy of age estimation is achieved.

$$
W=\left[\boldsymbol{u}_{1}, \boldsymbol{u}_{2}, \ldots, \boldsymbol{u}_{n}\right]
$$

Finally, the representation of input data in a new low-dimensional data space is calculated by Equation (11) using the transformation matrix $\boldsymbol{W}$ obtained with Equation (10).

$$
\boldsymbol{y}=\boldsymbol{W}^{T} \boldsymbol{x}
$$


The final step of our method for age estimation is to apply SVR on the age feature (y of Equation (10)) to estimate the age. Traditionally, the regression method is used to model the relationship between the input features and the ground-truth data. In order to obtain the best relationship between the age feature and the ground-truth age, we use the state-of-the-art method for regression, called SVR. Using training data, this method builds the relationship by choosing the type of kernel, its parameters, and some support vectors that fit the input feature data and ground-truth age. This model is saved and used for estimating the age of a new input image. For implementation, we used the LibSVM software package [41] in our experiments.

\section{Experimental Setup and Results}

\subsection{Database Description}

In our experiments, we use a public database for evaluating the performance of our system, called PAL database $[42,43]$. The updated PAL database contains the face images of 580 persons according to gender, facial expression (neutral, annoyed, happy, etc.), and race (Caucasians, Africa-Americans, and others). The age of the people used for the PAL database ranges from 18 to 93 years old. For each person, several images were captured with different types of facial expressions, including neutral and additional non-neutral expressions (happy, angry, annoyed, etc.). We randomly divided the PAL database into learning and testing databases twice (database parts 1 and 2, as listed in Table 3) in order to perform two-fold cross-validation to evaluate the performance of the age estimation system. At each trial, half of the images were assigned to the learning database, and the other half were assigned to the testing database. Images from the same person were not included in both the learning and testing databases. Detailed descriptions of the PAL database and the learning and testing databases used in our experiment are listed in Table 3. Some sample images from the PAL database are shown in Figure 11.

Table 3. Description of PAL database and division into sub-databases.

\begin{tabular}{llll}
\hline Database & Number of Learning Images & Number of Testing Images & Total \\
\hline Database Part 1 & 291 & 289 & 580 \\
Database Part 2 & 291 & 289 & 580 \\
\hline
\end{tabular}

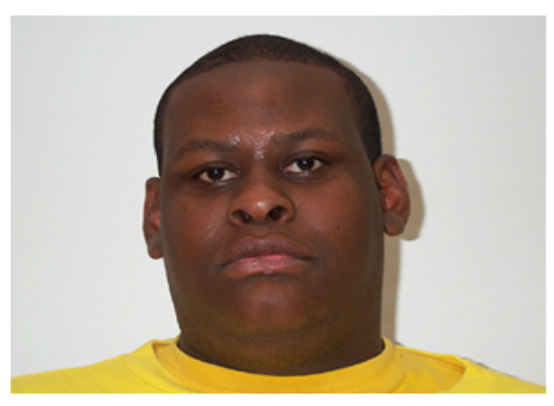

(a)

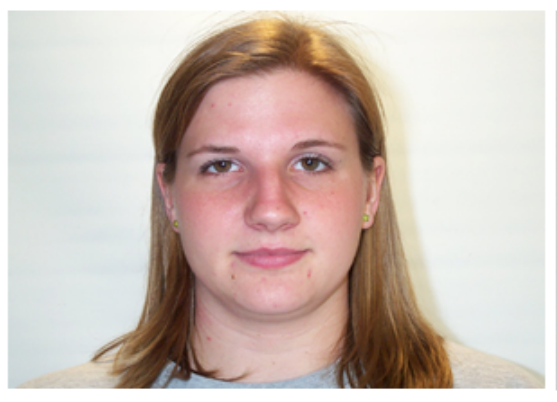

(b)

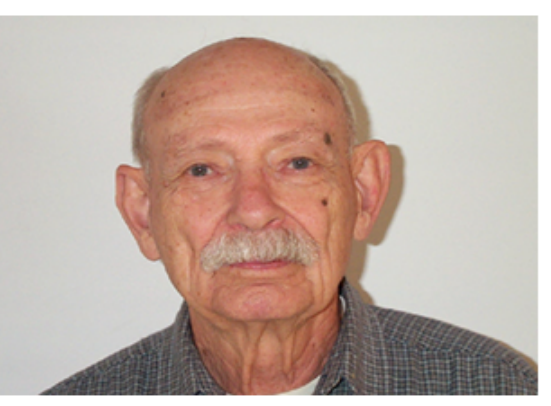

(c)

Figure 11. Examples of images in PAL database: (a) male age 22; (b) female age 21; and (c) male age 76 . 
There is no open database for age estimation that includes images with a blurring effect. Therefore, for our purpose of estimating age under the effects of image blurring, we created a blurring database from the original PAL database by applying the Gaussian blurring function to the original face images. The reason for using the Gaussian function is that PSF for optical blurring is usually defined as a Gaussian function [44]. In details, each original face image is blurred using five Gaussian kernels with different sigma values that range from one to five. With a larger sigma value of the Gaussian function, a more blurred image is obtained. Consequently, each person in the PAL database has six face images, including one original focused image and five blurred images. Therefore, we used a total of 3480 images (580 original images +2900 images generated by the five Gaussian kernels) for our experiments. As explained before, we divided these images into learning and testing databases twice (database parts 1 and 2 as listed in Table 4) in order to perform two-fold cross-validation to evaluate the performance of our age estimation system. From the six face images of each person, we classified three groups according to blurring degree, including the focused groups that contain the original image and the blurred image with sigma value one; the slightly blurred group that contains the blurred image with sigma 2; and the blurred group that contains the other blurred images with sigma from three to five. We performed various experiments based on the different cases of grouping with training data, and the optimal grouping determined by the best classification performance of the blurring images was achieved through our focus assessment method, as explained in Section 2.3 and Figure 5.

Table 4. Description (number of images) of the databases used in our experiments.

\begin{tabular}{ccccc}
\hline Database & $\begin{array}{c}\text { Focused Database } \\
\text { (DB1) }\end{array}$ & $\begin{array}{c}\text { Slightly Blurred } \\
\text { Database (DB2) }\end{array}$ & $\begin{array}{c}\text { Blurred } \\
\text { Database (DB3) }\end{array}$ & Total \\
\hline $\begin{array}{c}\text { Learning/Testing } \\
\text { (Database Part 1) }\end{array}$ & $582 / 578$ & $291 / 289$ & $873 / 867$ & $1746 / 1734$ \\
\hline $\begin{array}{l}\text { Learning/Testing } \\
\text { (Database Part 2) }\end{array}$ & $582 / 578$ & $291 / 289$ & $873 / 867$ & $1746 / 1734$ \\
\hline
\end{tabular}

Finally, we obtained the blurred databases that correspond to the three groups (DB1, DB2, and DB3) according to blurring degree as listed in Table 4. In Figure 12, we show some sample images according to the sigma value of the Gaussian blurring function.

MAE is used widely as the criterion for measuring the accuracy of age estimation systems $[1,13,14,30]$, and we use MAE in our experiments. Mathematically, MAE is the measure of the average error of the estimated ages $\left(a_{k}\right)$ compared with the ground-truth ages $\left(a_{k}{ }^{\prime}\right)$ as shown in Equation (12) [14,30].

$$
M A E=\frac{1}{N} \sum_{k=1}^{N}\left|a_{k}{ }^{\prime}-a_{k}\right|
$$

In Equation (12), $N$ is the number of testing data. A smaller value of MAE shows better estimation accuracy of the age estimation system. 

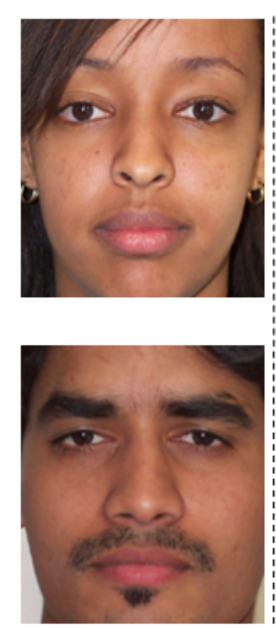

$\sigma=0$

(Original Image)
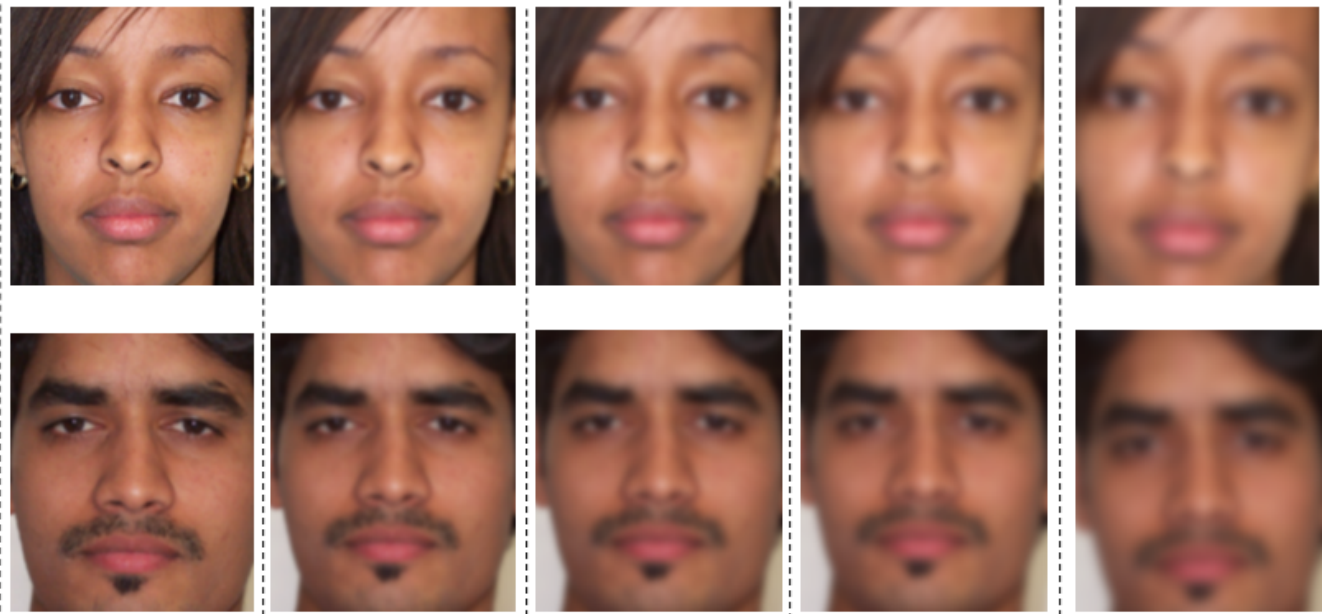

$\sigma=1$

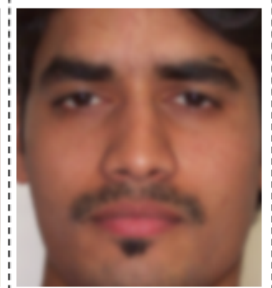

$\sigma=2$

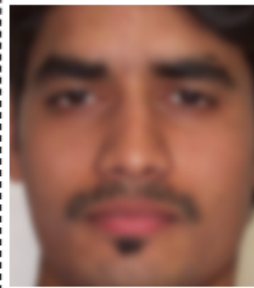

$\sigma=3$

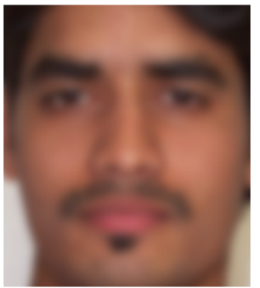

$\sigma=4$

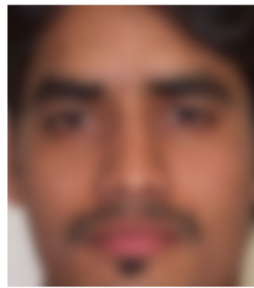

$\sigma=5$

Figure 12. Examples of original and blurred face images produced by the Gaussian blurring function at various sigma values $(\sigma)$ of the Gaussian function.

\subsection{Performance Evaluation of Previous Age Estimation Systems}

In the first experiment, we evaluated the performance of previous age estimation systems $[13,14,16]$. We used the PAL databases described in Table 3 for this experiment. Detailed estimation performance (MAE) for this experiment is indicated in Table 5. The results indicate that the combined method of MLBP and Gabor [14] produces better estimation results compared with other methods.

Table 5. Comparison of the performance (MAE) of previous age estimation systems with the PAL database (unit: years).

\begin{tabular}{llll}
\hline Database & $\begin{array}{l}\text { MLBP-Based } \\
\text { Method [16] }\end{array}$ & $\begin{array}{l}\text { Gabor-Based } \\
\text { Method }\end{array}$ & $\begin{array}{l}\text { Combination of MLBP and } \\
\text { Gabor-Based Methods [14] }\end{array}$ \\
\hline Testing Database 1 & 6.457 & 11.803 & 6.339 \\
Testing Database 2 & 6.581 & 12.208 & 6.498 \\
Average & 6.519 & 12.006 & 6.419 \\
\hline Previous Research [13] & 8.44 & & \\
\hline
\end{tabular}

For the next experiment, we evaluate performance under the effects of image blurring on previous age estimation systems. The experiment results are listed in Table 6. As observed from the table, we find that the estimation error increases significantly with blurred images compared with focused images. In details, using only focused images (original PAL database), the lowest MAE of 6.419 years was obtained. However, when the images are blurred, MAEs increased significantly at 7.715 years with DB1 (focused images + very slightly blurred image), 19.914 years with DB2 (slightly blurred images), and 21.738 years with DB3 (significantly blurred images). The descriptions for DB1, DB2, and DB3 are listed in Table 4. On average, MAE increases from 6.419 years with the focused database to 16.279 years with the blurred database. From this experiment result, we can conclude that the blurring of input images has extremely strong effects on age estimation systems. 
Table 6. Comparisons of the performance (MAE) of previous age estimation systems with the blurred images (Average means the average MAE with DB1, DB2, and DB3) (unit: years).

\begin{tabular}{llllll}
\hline Methods & $\begin{array}{l}\text { Original PAL } \\
\text { Database } \\
(\boldsymbol{\sigma}=\mathbf{0})\end{array}$ & $\begin{array}{l}\text { Focused Database } \\
(\mathbf{D B 1})(\boldsymbol{\sigma}=\mathbf{0 , 1})\end{array}$ & $\begin{array}{l}\text { Slightly Blurred } \\
\text { Database } \\
(\mathbf{\sigma}=\mathbf{2})\end{array}$ & $\begin{array}{l}\text { Blurred } \\
\text { Database (DB3) }\end{array}$ & $\begin{array}{l}\text { Average } \\
(\boldsymbol{\sigma}=\mathbf{3 , 4 , 5})\end{array}$ \\
\hline MLBP-based Method [16] & 6.519 & 8.220 & 20.229 & 22.813 & 17.535 \\
\hline Gabor-based Method & 12.006 & 15.436 & 20.689 & 22.847 & 20.010 \\
\hline $\begin{array}{l}\text { Combination of MLBP and } \\
\text { Gabor-based Methods [14] }\end{array}$ & 6.419 & 7.715 & 19.914 & 21.738 & $\mathbf{1 6 . 2 7 9}$ \\
\hline
\end{tabular}

\subsection{Performance Evaluation of Our Method}

In this section, we show the experiment results of the performance of our age estimation system. As explained in Section 2.4, one of the problems of the previous age estimation system [14] based on a combination of MLBP, Gabor filtering, and SVR is the high dimension of the age feature. This problem can be solved by applying the PCA method on the extracted feature before inputting to SVR in order to estimate human age. To evaluate the efficiency of the PCA method on feature dimension reduction and performance enhancement, we measured the estimation performance (MAE) of the age estimation system with and without the PCA method. Because the focused database (DB1) contains both the original focused PAL images and very slightly blurred images (blurred images with $\sigma$ one), in this experiment, we measure the performance of the age estimation system with and without PCA for three blurred databases, including DB1, DB2, and DB3, as listed in Table 7. As indicated in this table, the system that does not apply the PCA method produces MAE of 7.194 years. With PCA, MAE is reduced to 6.845 years. In details, all the MAE values for DB1, DB2, and DB3 with the PCA method are reduced compared with those without the PCA method. Through these experiment results, the PCA method is proven to be useful for reducing feature dimension and enhancing the performance of age estimation systems. In Table 7 , the pre-classification of DB1, DB2, and DB3 is performed manually because we want to check only the effects of feature extraction by PCA on the performance of age estimation.

Table 7. Estimation performance (MAE) of the age estimation system with and without applying PCA using three databases, including DB1, DB2, and DB3 (unit: years).

\begin{tabular}{lllll}
\hline \multirow{2}{*}{ Database } & \multicolumn{3}{c}{ With PCA } & Without \\
\cline { 2 - 4 } & Testing Database 1 & Testing Database 2 & Average & PCA \\
\hline DB1 & 6.114 & 6.420 & 6.267 & 6.630 \\
DB2 & 6.882 & 6.900 & 6.891 & 7.240 \\
DB3 & 7.173 & 7.257 & 7.215 & 7.556 \\
Average with Entire Database & 6.771 & 6.918 & $\mathbf{6 . 8 4 5}$ & $\mathbf{7 . 1 9 4}$ \\
\hline
\end{tabular}

For the next experiment, we evaluate the performance of the classification of blurred images into blurring groups using the MLBP measurement as explained in Section 2.3 and Figure 5. For this purpose, we use the three blurred databases (DB1, DB2, and DB3). As explained in Section 3.1, DB1 contains the images of the PAL database and very slightly blurred images with sigma value one. This characteristic causes high FSs of images in DB1 (close to 100). DB2 contains the blurred images of the images in the 
PAL database with sigma value two. This database represents the class of slightly blurred images. Therefore, the FSs of the images in this database are expected to be lower than those for DB1. Finally, DB3 contains the blurred images of the images in the PAL database with larger sigma values (sigma values three, four, and five). The images in this database are blurred significantly, as shown in Figure 12. Consequently, the FSs of the images in the DB3 database are expected to be extremely small.

As explained in Section 2.3 and Figure 5, in order to classify the images into one of the groups according to the blurring degree, we determine the classification thresholds where misclassification is smallest. The classification results that use two-fold cross-validation with the testing databases are provided in Table 8. Note that because the blurring degree of DB3 is too high, two images in the testing database of DB3 failed for face detection. Consequently, in Table 8, the total number of images in DB3 is 865 , instead of 867 , as indicated in Table 4. Using the thresholds obtained from the learning databases, the classification results for the testing database are provided in Table 8. On average, the correct classification of the testing database is approximately $97.69 \%$. From these results, we conclude that our focus assessment method can be used for classification of image blurring.

Table 8. Classification results when classifying the input images into one of three groups according to blurring degree for the testing databases.

\begin{tabular}{|c|c|c|c|c|c|c|c|c|}
\hline \multirow{2}{*}{\multicolumn{2}{|c|}{$\begin{array}{l}\text { Number of Images } \\
\text { (Classification Rate) }\end{array}$}} & \multirow{2}{*}{$\begin{array}{c}\text { Correct } \\
\text { Classification } \\
\end{array}$} & \multicolumn{6}{|c|}{ Incorrect Classification } \\
\hline & & & DB1 $\rightarrow$ DB2 & $\mathrm{DB} 2 \rightarrow \mathrm{DB} 1$ & DB1 $\rightarrow$ DB3 & DB3 $\rightarrow$ DB1 & DB2 $\rightarrow$ DB3 & DB3 $\rightarrow$ DB2 \\
\hline \multirow{2}{*}{ DB1 } & $\begin{array}{c}\text { Testing } \\
\text { Database } 1 \\
\end{array}$ & $576(99.65 \%)$ & $2(0.35 \%)$ & - & $0(0 \%)$ & - & - & - \\
\hline & $\begin{array}{c}\text { Testing } \\
\text { Database } 2\end{array}$ & $576(99.65 \%)$ & $2(0.35 \%)$ & - & $0(0 \%)$ & - & - & - \\
\hline \multirow{2}{*}{ DB2 } & $\begin{array}{c}\text { Testing } \\
\text { Database } 1\end{array}$ & $267(92.39 \%)$ & - & $5(1.73 \%)$ & - & - & $17(5.88 \%)$ & - \\
\hline & $\begin{array}{c}\text { Testing } \\
\text { Database } 2\end{array}$ & $256(88.58 \%)$ & - & $12(4.15 \%)$ & - & - & $21(7.27 \%)$ & - \\
\hline \multirow{2}{*}{ DB3 } & $\begin{array}{c}\text { Testing } \\
\text { Database } 1\end{array}$ & $856(98.96 \%)$ & - & - & - & $0(0 \%)$ & - & $9(1.04 \%)$ \\
\hline & $\begin{array}{c}\text { Testing } \\
\text { Database } 2\end{array}$ & $853(98.61 \%)$ & - & - & - & $0(0 \%)$ & - & $12(1.39 \%)$ \\
\hline
\end{tabular}

As explained in Section 2.3, our method uses the age estimator optimized by the corresponding data of each group. Therefore, the misclassification error in Table 8 can cause an error in age estimation. Therefore, in order to reduce the effects of the misclassified images from Table 8, we classify the face images into one of the five groups according to blurring degree. Among the five groups in Figure 5, the two groups called $G_{2}$ and $G_{4}$ are the overlapped groups. If the images are determined as belonging to the overlapped groups $G_{2}$ and $G_{4}$, the age of the face image is obtained by combining the two estimated ages by the age estimators of the two neighboring groups. For example, if the FS of an image belongs to $G_{4}$, the final age of the face image is obtained by combining the two estimated ages from the two age estimators $A E_{3}$ and $A E_{5}$. We performed the classification into one of the five groups with the testing database, and the results are listed in Table 9. Because two images in DB3 failed for face detection as a result of excessive blurring, the total number of images in the testing database is 1732 (instead of 1734 images as 
shown in Table 4). In the experiment indicated in Table 9, the entire data for DB1, DB2, and DB3 are used.

Table 9. Classification results of the images placed into one of the five groups according to blurring degree and considering overlapped groups.

\begin{tabular}{lllllll}
\hline Number of Images & $\mathbf{G}_{\mathbf{1}}$ & $\mathbf{G}_{\mathbf{2}}$ & $\mathbf{G}_{\mathbf{3}}$ & $\mathbf{G}_{\mathbf{4}}$ & $\mathbf{G}_{\mathbf{5}}$ & Total \\
\hline Testing Database 1 & 660 & 348 & 108 & 61 & 555 & 1732 \\
Testing Database 2 & 656 & 359 & 95 & 69 & 553 & 1732 \\
\hline
\end{tabular}

For the images in the three groups, including $G_{1}, G_{3}$, and $G_{5}$ of Figure 5, age is estimated directly using the age estimators $A E_{1}, A E_{3}$, and $A E_{5}$, respectively. For the images in the other two groups, $G_{2}$ and $G_{4}$, because we employed two neighborhood age estimators to estimate the age, the final estimated age of the image is obtained by the combination of the two estimated ages.

For our experiments, we compare the accuracies of age estimation using three different combination methods, including MIN, MAX, and SUM rule. In details, suppose that using the two neighboring age estimators, we obtain the estimated age of a face image in group $G_{2}$ (or $G_{4}$ ) as $A g e_{1}$ and $A g e_{2}$. The MIN rule indicates that the combined age is produced by taking the minimum value of the two estimated ages. Similarly, the MAX rule takes the maximum value of two estimated ages as the combined age. Finally, the age estimated by the SUM rule is calculated by the average value of the two age values. The formula for these rules can be expressed in Equations (13) to (15) as follows:

$$
\begin{aligned}
& \text { MIN Rule: } A G E=M I N\left(A g e_{1}, A g e_{2}\right) \\
& \text { MAX Rule: } A G E=M A X\left(A g e_{1}, A g e_{2}\right) \\
& \text { SUM Rule: } A G E=\left(A g e_{1}+A g e_{2}\right) / 2
\end{aligned}
$$

The experiment results of these experiments are provided in Tables 10-12. As indicated in these tables, the best estimation performance is obtained using the SUM rule, and the estimated MAE with the entire database (DB1, DB2, and DB3) is 6.91 years. If no error occurs in the blurred image classification steps, MAE for the age estimation system is 6.845 years, as indicated in Table 7. However, in real systems, errors can occur. Therefore, the performance of the age estimation system can degrade. From the two estimation results, we can see that, although some errors occurred during the blurred image classification step, the performance of the age estimation system is reduced slightly (6.91 years versus 6.845 years). Compared with the results of the previous methods listed in Table 6 , we can conclude that our method is more efficient at making age estimation systems robust to blurring effects, compared with previous methods $[14,16]$.

Table 10. Performance of our method using MIN rule on $\mathrm{G}_{2}$ and $\mathrm{G}_{4}$ groups (unit: years).

\begin{tabular}{llllll}
\hline MAE & $\mathbf{G}_{\mathbf{1}}$ & $\mathbf{G}_{\mathbf{2}}$ & $\mathbf{G}_{\mathbf{3}}$ & $\mathbf{G}_{\mathbf{4}}$ & $\mathbf{G}_{\mathbf{5}}$ \\
\hline Testing Database 1 & 7.423 & 8.620 & 6.083 & 6.459 & 6.083 \\
Testing Database 2 & 7.206 & 8.652 & 6.400 & 5.797 & 6.452 \\
Average & 7.315 & 8.636 & 6.231 & 6.108 & 6.267 \\
\hline Average MAE with the Entire Database & & \multicolumn{5}{c}{7.141} \\
\hline
\end{tabular}


Table 11. Performance of our method using $\mathrm{MAX}$ rule on $\mathrm{G}_{2}$ and $\mathrm{G}_{4}$ groups (unit: years).

\begin{tabular}{llllll}
\hline MAE & $\mathbf{G}_{\mathbf{1}}$ & $\mathbf{G}_{\mathbf{2}}$ & $\mathbf{G}_{\mathbf{3}}$ & $\mathbf{G}_{\mathbf{4}}$ & $\mathbf{G}_{\mathbf{5}}$ \\
\hline Testing Database 1 & 7.423 & 7.704 & 6.083 & 13.279 & 6.083 \\
Testing Database 2 & 7.206 & 7.903 & 6.400 & 11.957 & 6.452 \\
Average & 7.315 & 7.805 & 6.231 & 12.577 & 6.267 \\
\hline Average MAE with the Entire Database & & \multicolumn{3}{c}{7.214} \\
\hline
\end{tabular}

Table 12. Performance of our method using SUM rule on $\mathrm{G}_{2}$ and $\mathrm{G}_{4}$ groups (unit: years).

\begin{tabular}{llllll}
\hline MAE & $\mathbf{G}_{\mathbf{1}}$ & $\mathbf{G}_{\mathbf{2}}$ & $\mathbf{G}_{\mathbf{3}}$ & $\mathbf{G}_{\mathbf{4}}$ & $\mathbf{G}_{\mathbf{5}}$ \\
\hline Testing Database 1 & 7.423 & 7.319 & 6.083 & 8.574 & 6.083 \\
Testing Database 2 & 7.206 & 6.797 & 6.400 & 8.580 & 6.452 \\
Average & 7.315 & 7.054 & 6.231 & 8.577 & 6.267 \\
\hline Average MAE with the Entire Database & \multicolumn{5}{c}{6.91} \\
\hline
\end{tabular}

In Figure 13, we show some examples of the age estimation results obtained through the proposed and previous methods [14]. In these examples, "Estimated age_1" and "Estimated age_2" indicate the ages estimated using the proposed and previous methods [14], respectively. The estimation error is smaller with the system that uses our method. It can be observed from these examples that, although the images are focused (Figure 13a), slightly blurred (Figure 13b,c), or significantly blurred (Figure 13d-f), the estimation results through our method are still good. Therefore, we can conclude that our method is efficient for estimating age of human in images at different blurring degrees.

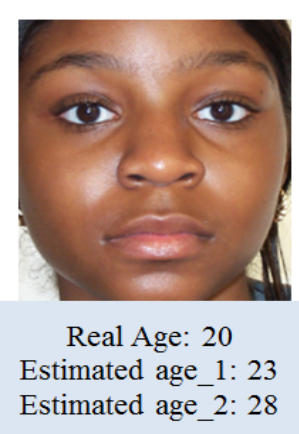

(a)

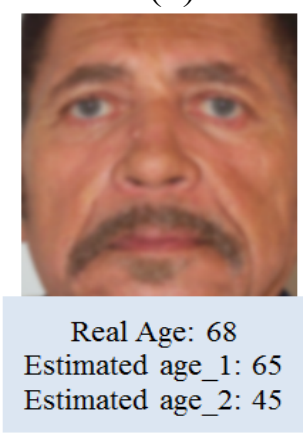

(d)

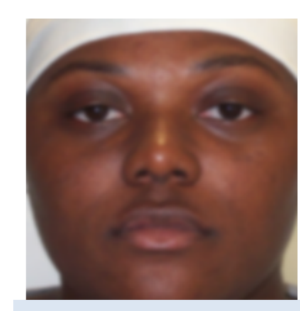

Real Age: 22 Estimated age_1: 22 Estimated age_2: 33

(b)

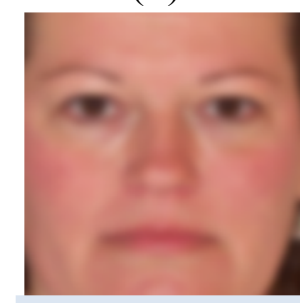

Real Age: 42 Estimated age_1: 45 Estimated age_2: 29

(e)

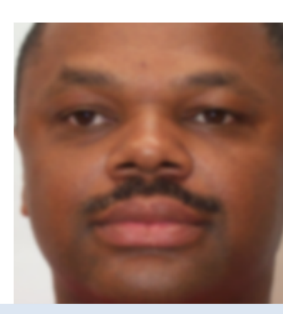

Real Age: 39 Estimated age_1: 38 Estimated age_2: 30

(c)

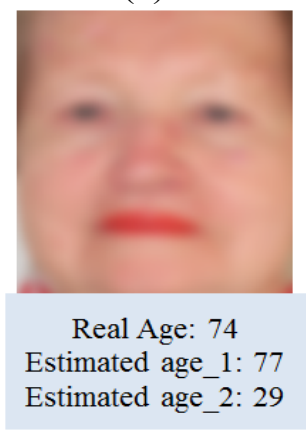

(f)

Figure 13. Comparisons of age estimation results with the proposed (using SUM rule) and previous methods [14]. 
In Figure 14, we show some examples of age estimation results of images which are incorrectly classified into one of the blurring group $\left(G_{1}, \ldots, G_{5}\right.$ of Figure 5$)$ using FS. As shown in Figure 14a-d, the misclassification of images into one of the blurring group causes the significantly errors on estimation results. For example of Figure 14a, because it is misclassified into $G_{3}$ of Figure 5, the estimated age by $A E_{3}$ becomes 63, and the error between the real and estimated ages is large as 30 (63-33). However, by using our method considering the overlapped area of Figure 5, the final estimated age comes to 47 based on the SUM rule of Equation (15) with two estimated ages by $A E_{5}(30)$ and $A E_{3}$ (63), and the consequent error of the age estimation is reduced as 14 (47-33). Like the Figure 14a, in other cases of Figure 14b-d, we can reduce the error of the age estimation caused by the misclassification of images into one of the blurring group based on our method. In addition, through these examples, we can find that the blurring effect has significantly effects on age estimation.

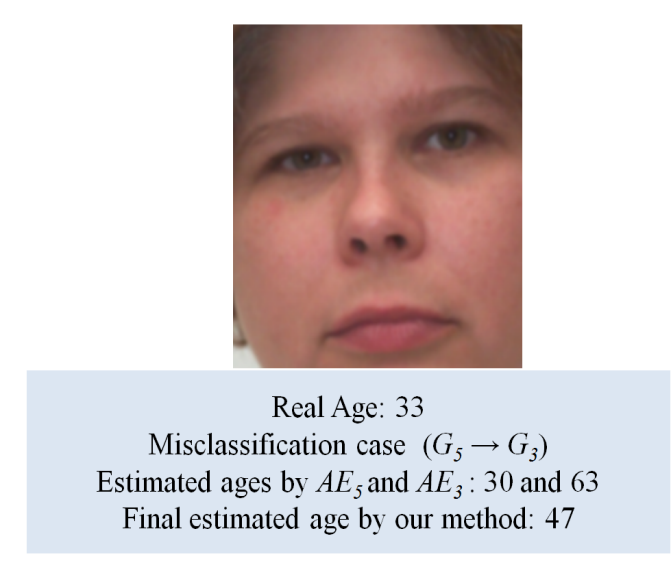

(a)

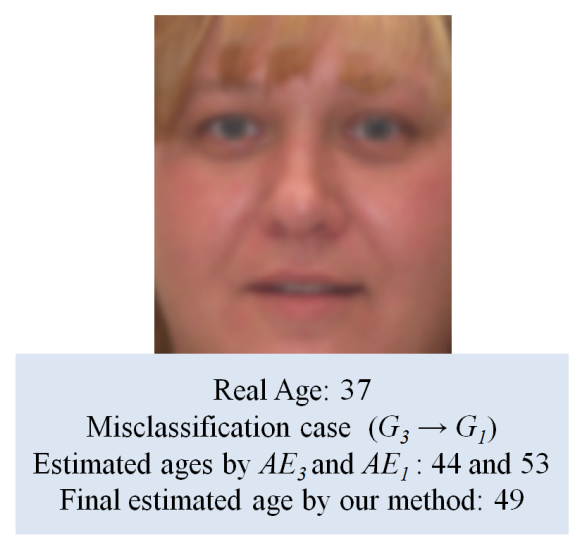

(c)

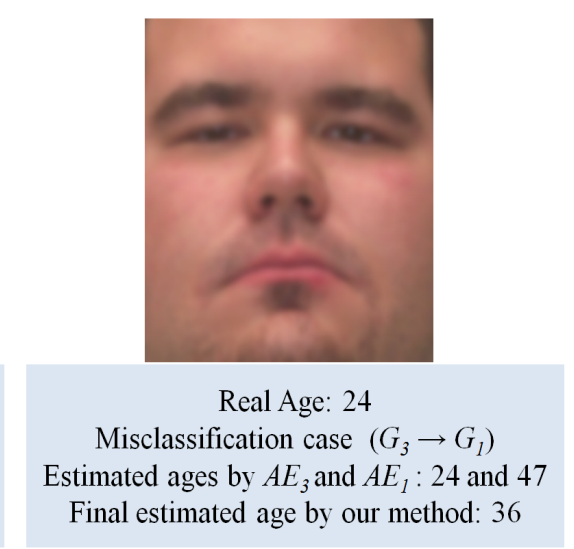

(b)

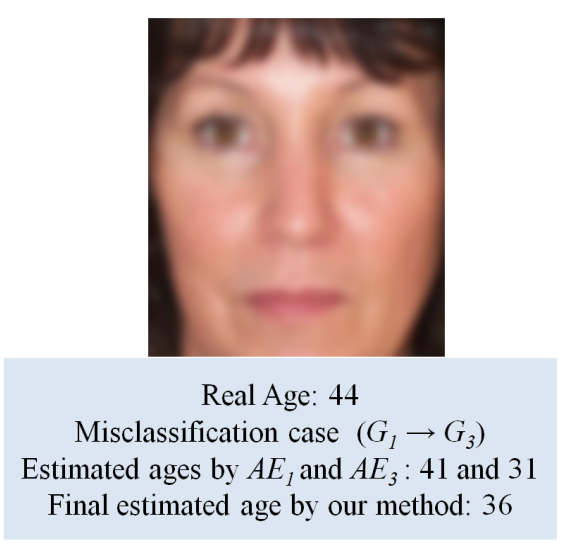

(d)

Figure 14. Examples of age estimation results with misclassification of blurring group, and the accuracy enhancement by our method with these error cases: misclassification of (a) $G_{5} \rightarrow G_{3}$; (b,c) $G_{3} \rightarrow G_{1}$; and (d) $G_{1} \rightarrow G_{3}$.

As the next experiments, we repeat the experiments using another dataset. For this purpose, we use the MORPH database in the new experiments [45]. The MORPH database (album 2) contains 55,134 facial images of 13,617 individuals. From this database, we randomly select 1000 images at different ages, genders and individuals for our new experiments. The description of the selected database is given in Table 13. Totally, we perform experiments using 6000 images (1000 original focused images and 5000 optical 
blurring images), including 3000 images for training and 3000 images for testing. We repeat the experiments twice to perform two-fold cross-validation scheme. Like the previous experiments on PAL database, we divide the optical blurring into three sub-databases, including focused database (DB4), slightly database (DB5) and blurred database (DB6) as shown in Table 13. The detail experiment results of the new experiments are shown from Tables 14-20. As shown in Table 14, without optical blurring effects, the estimation performance (MAE) using the MORPH database is about 6.254 years. In Table 15, by presenting the optical blurring on face images, the MAE becomes to 9.026 years that is much higher than 6.254 years in system without optical blurring effects. This result, one again, confirms that the optical blurring has very strong effects on age estimation system. By using our proposed method, the MAE is reduced from 9.026 years to 6.607 years using SUM rule, as shown in Table 20, which is similar to that (6.91) with PAL database in Table 12. From this result, we can conclude that our proposed method is sufficient for age estimation system robust to optical blurring effects. In addition, Table 16 gives the classification results of blurred images into three groups of focused, slightly blurred and blurred group. On average, we obtain the classification accuracy of $97.217 \%$, which is similar to that $(97.69 \%)$ with PAL database in Table 8.

These results show that the performances by our method are less affected by the kinds of databases.

Table 13. Description of the selected database.

\begin{tabular}{ccccc}
\hline Database & $\begin{array}{c}\text { Focused } \\
\text { Database (DB4) }\end{array}$ & $\begin{array}{c}\text { Slightly Blurred } \\
\text { Database (DB5) }\end{array}$ & $\begin{array}{c}\text { Blurred } \\
\text { Database (DB6) }\end{array}$ & $\begin{array}{c}\text { Total Number } \\
\text { of Images }\end{array}$ \\
\hline $\begin{array}{l}\text { Learning/Testing } \\
\text { (Database Part 1) }\end{array}$ & $1000 / 1000$ & $500 / 500$ & $1500 / 1500$ & $3000 / 3000$ \\
\hline $\begin{array}{l}\text { Learning/Testing } \\
\text { (Database Part 2) }\end{array}$ & $1000 / 1000$ & $500 / 500$ & $1500 / 1500$ & $3000 / 3000$ \\
\hline
\end{tabular}

Table 14. Estimation performance using original selected database (unit: years old).

\begin{tabular}{ccc}
\hline Testing Database 1 & Testing Database 2 & Average MAE \\
\hline 6.162 & 6.346 & 6.254 \\
\hline
\end{tabular}

Table 15. Comparisons of the performance (MAE) of previous age estimation system with the blurred images (unit: years).

\begin{tabular}{ccccc}
\hline MAE & $\begin{array}{c}\text { Original Selected } \\
\text { Database }(\boldsymbol{\sigma}=\mathbf{0})\end{array}$ & $\begin{array}{c}\text { Focused Database } \\
(\boldsymbol{\sigma}=\mathbf{0 , 1})\end{array}$ & $\begin{array}{c}\text { Slightly Blurred } \\
\text { Database }(\boldsymbol{\sigma}=\mathbf{2})\end{array}$ & $\begin{array}{c}\text { Blurred Database } \\
(\boldsymbol{\sigma}=\mathbf{3 , 4 , 5})\end{array}$ \\
\hline Testing Database 1 & 6.162 & 6.621 & 8.600 & 10.577 \\
Testing Database 2 & 6.346 & 6.757 & 8.882 & 10.781 \\
\hline \multirow{2}{*}{ Average MAE } & 6.254 & 6.689 & 8.741 & 10.679 \\
\cline { 3 - 5 } & & & $\mathbf{9 . 0 2 6}$ & \\
\hline
\end{tabular}


Table 16. Classification results when classifying the input images into one of three groups according to blurring degree for testing databases.

\begin{tabular}{|c|c|c|c|c|c|c|c|c|}
\hline \multirow{2}{*}{\multicolumn{2}{|c|}{$\begin{array}{l}\text { Number of Images } \\
\text { (Classification Rate) }\end{array}$}} & \multirow{2}{*}{$\begin{array}{c}\text { Correct } \\
\text { Classification } \\
\end{array}$} & \multicolumn{6}{|c|}{ Incorrect Classification } \\
\hline & & & DB4 $\rightarrow$ DB5 & DB5 $\rightarrow$ DB4 & DB4 $\rightarrow$ DB6 & DB6 $\rightarrow$ DB4 & DB5 $\rightarrow$ DB6 & DB6 $\rightarrow$ DB5 \\
\hline \multirow{2}{*}{ DB4 } & $\begin{array}{c}\text { Testing } \\
\text { Database } 1\end{array}$ & $979(97.9 \%)$ & $21(2.1 \%)$ & - & $0(0.0 \%)$ & - & - & - \\
\hline & $\begin{array}{c}\text { Testing } \\
\text { Database } 2\end{array}$ & $982(98.2 \%)$ & $18(1.8 \%)$ & - & $0(0.0 \%)$ & - & - & - \\
\hline \multirow{2}{*}{ DB5 } & $\begin{array}{c}\text { Testing } \\
\text { Database } 1\end{array}$ & $443(88.6 \%)$ & - & $35(7.0 \%)$ & - & - & $22(4.4 \%)$ & - \\
\hline & $\begin{array}{c}\text { Testing } \\
\text { Database } 2 \\
\end{array}$ & $450(90.0 \%)$ & - & $29(5.8 \%)$ & - & - & $21(4.2 \%)$ & - \\
\hline \multirow{2}{*}{ DB6 } & $\begin{array}{c}\text { Testing } \\
\text { Database } 1\end{array}$ & $1488(99.2 \%)$ & - & - & - & $0(0.0 \%)$ & - & $12(0.8 \%)$ \\
\hline & $\begin{array}{c}\text { Testing } \\
\text { Database } 2\end{array}$ & $1491(99.4 \%)$ & - & - & - & $0(0.0 \%)$ & - & $9(0.6 \%)$ \\
\hline
\end{tabular}

Table 17. Classification results of the images placed into one of five groups according to blurring degree and considering overlapped groups.

\begin{tabular}{ccccccc}
\hline Number of Images & $\mathbf{G}_{\mathbf{1}}$ & $\mathbf{G}_{\mathbf{2}}$ & $\mathbf{G}_{\mathbf{3}}$ & $\mathbf{G}_{\mathbf{4}}$ & $\mathbf{G}_{\mathbf{5}}$ & Total Number of Images \\
\hline Testing Database 1 & 1361 & 165 & 332 & 199 & 943 & 3000 \\
Testing Database 2 & 1360 & 164 & 330 & 204 & 942 & 3000 \\
\hline
\end{tabular}

Table 18. Performance of our proposed method using MIN rule on G2 and G4 groups (unit: years).

\begin{tabular}{cccccc}
\hline MAE & $\mathbf{G}_{\mathbf{1}}$ & $\mathbf{G}_{\mathbf{2}}$ & $\mathbf{G}_{\mathbf{3}}$ & $\mathbf{G}_{\mathbf{4}}$ & $\mathbf{G}_{\mathbf{5}}$ \\
\hline Testing Database 1 & 7.182 & 7.079 & 6.557 & 6.744 & 6.098 \\
Testing Database 2 & 6.865 & 6.829 & 6.427 & 7.025 & 6.088 \\
Average & 7.024 & 6.954 & 6.492 & 6.886 & 6.093 \\
\hline Average MAE with the Entire Database & & & 6.660 & & \\
\hline
\end{tabular}

Table 19. Performance of our proposed method using MAX rule on G2 and G4 groups (unit: years).

\begin{tabular}{cccccc}
\hline MAE & $\mathbf{G}_{\mathbf{1}}$ & $\mathbf{G}_{\mathbf{2}}$ & $\mathbf{G}_{\mathbf{3}}$ & $\mathbf{G}_{\mathbf{4}}$ & $\mathbf{G}_{\mathbf{5}}$ \\
\hline Testing Database 1 & 7.182 & 6.824 & 6.557 & 7.015 & 6.098 \\
Testing Database 2 & 6.865 & 6.927 & 6.427 & 6.990 & 6.088 \\
Average & 7.024 & 6.875 & 6.492 & 7.002 & 6.093 \\
\hline Average MAE with the Entire Database & & & 6.663 & & \\
\hline
\end{tabular}


Table 20. Performance of our proposed method using SUM rule on G2 and G4 groups (unit: years).

\begin{tabular}{cccccc}
\hline MAE & $\mathbf{G}_{\mathbf{1}}$ & $\mathbf{G}_{\mathbf{2}}$ & $\mathbf{G}_{\mathbf{3}}$ & $\mathbf{G}_{\mathbf{4}}$ & $\mathbf{G}_{\mathbf{5}}$ \\
\hline Testing Database 1 & 7.182 & 6.552 & 6.557 & 6.302 & 6.098 \\
Testing Database 2 & 6.865 & 6.634 & 6.427 & 6.485 & 6.088 \\
Average & 7.024 & 6.593 & 6.492 & 6.395 & 6.093 \\
\hline Average MAE with the Entire Database & & & $\mathbf{6 . 6 0 7}$ & & \\
\hline
\end{tabular}

We show some examples estimation results with MORPH database in the Figure 15. Similar to Figure 13, our proposed method produces the better estimation result compared to previous method [14]. In this figure, "Estimated age_1" and "Estimated age_2" indicate the ages estimated using the proposed and previous methods [14], respectively. In addition, Figure 16 shows some examples of estimation results of images in overlapped area $\left(\mathrm{G}_{2}\right.$ and $\left.\mathrm{G}_{4}\right)$. By taking the average estimation results of two neighbor groups, the final estimation results of images in overlapped areas are enhanced compared to estimation results of system without considering the overlapped areas.

Finally, in order to confirm the effects of optical blurring on age estimation systems, we performed additional experiments using an open age estimation program, called OpenBR [46]. For this purpose, we used all the blurred images of PAL database with the sigma value of Gaussian blurring kernel from 1 to 5, as shown Table 4. The consequent experiment results of these experiments are given by Table 21. As shown in the table, the average MAE using the OpenBR-age-estimation method was about 14.35 years. Compared to our method, which produced MAE of 6.91 years (Table 12), we can conclude that our method outperforms the OpenBR-age-estimation method on age estimation problem. In addition, the MAEs in Table 21 are increased according to the degree of optical blurring (the sigma value of Gaussian blurring). This result demonstrates that the optical blurring has strong effects on the age estimation system.

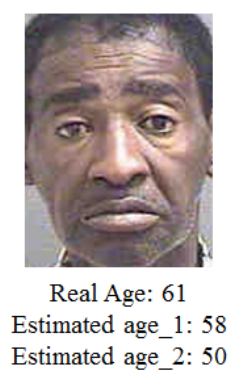

(a)

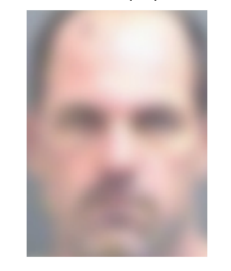

Real Age: 42 Estimated age_1: 43 Estimated age_2: 32

(e)

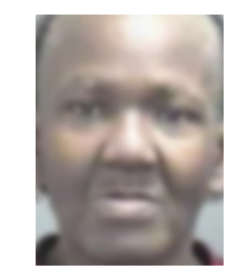

Real Age: 54 Estimated age_1: 55 Estimated age_2: 41

(b)

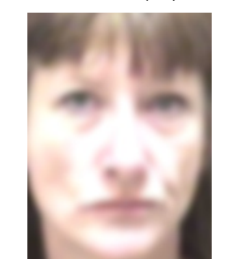

Real Age: 47

Estimated age_1: 47

Estimated age_2: 35

(f)

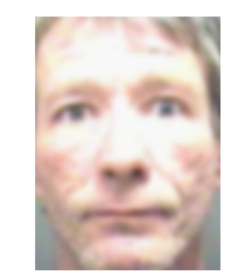

Real Age: 48

Estimated age_1: 46

Estimated age_2: 37

(c)

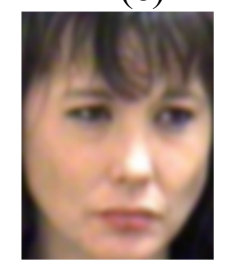

Real Age: 36

Estimated age_1: 33

Estimated age_2: 30

(g)

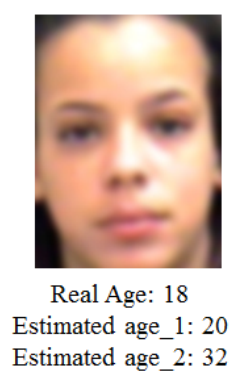

(d)

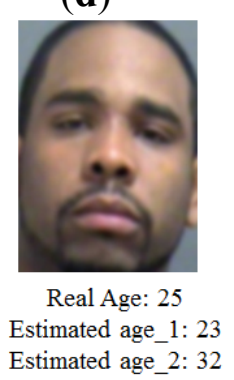

(h)

Figure 15. Comparisons of age estimation results with the proposed (using SUM rule) and previous method [14] using MORPH database. 


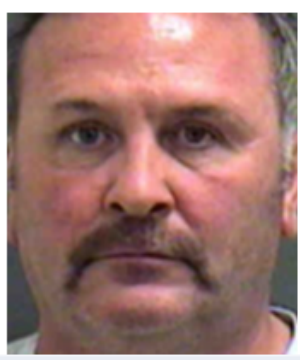

Real Age: 45

Misclassification case $(\mathrm{G} 5 \rightarrow \mathrm{G} 3$ )

Estimated by $\mathrm{AE}_{5}$ and $\mathrm{AE}_{3}: 50$ and 60

Final estimated age by our method: 55

(a)

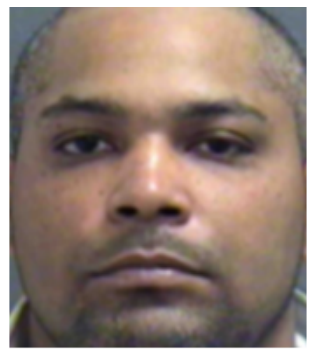

Real Age: 31

Misclassification case $(\mathrm{G} 3 \rightarrow \mathrm{G} 1)$

Estimated by $\mathrm{AE}_{3}$ and $\mathrm{AE}_{1}: 31$ and 43

Final estimated age by our method: 37

(c)

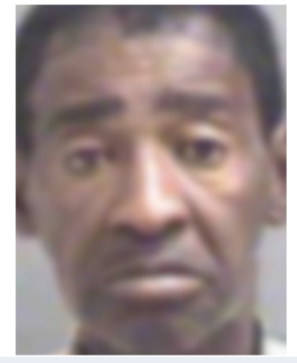

Real Age: 61

Misclassification case $(\mathrm{G} 3 \rightarrow \mathrm{G} 5)$ Estimated by $\mathrm{AE}_{3}$ and $\mathrm{AE}_{5}$ : 59 and 52 Final estimated age by our method: 56

(b)

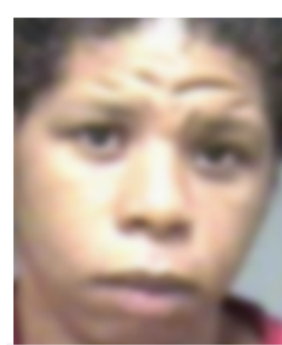

Real Age: 32

Misclassification case $(\mathrm{G} 1 \rightarrow \mathrm{G} 3$ )

Estimated by $\mathrm{AE}_{1}$ and $\mathrm{AE}_{3}: 34$ and 39

Final estimated age by our method: 37

(d)

Figure 16. Examples of age estimation results with misclassification of blurring group, and the accuracy enhancement by our method with these error cases: misclassification of (a) $\mathrm{G}_{5} \rightarrow \mathrm{G}_{3}$; (b) $\mathrm{G}_{3} \rightarrow \mathrm{G}_{5}$; (c) $\mathrm{G}_{3} \rightarrow \mathrm{G}_{1}$; and (d) $\mathrm{G}_{1} \rightarrow \mathrm{G}_{3}$.

Table 21. The estimation result (MAE) of OpenBR-age-estimation method using blurred PAL database (unit: years).

\begin{tabular}{cccccc}
\hline MAE & $\begin{array}{c}\text { Blurred Images } \\
\text { with Sigma of } \mathbf{1}\end{array}$ & $\begin{array}{c}\text { Blurred Images } \\
\text { with Sigma of 2 }\end{array}$ & $\begin{array}{c}\text { Blurred Images } \\
\text { with Sigma of 3 }\end{array}$ & $\begin{array}{c}\text { Blurred Images } \\
\text { with Sigma of 4 }\end{array}$ & $\begin{array}{c}\text { Blurred Images } \\
\text { with Sigma of 5 }\end{array}$ \\
\hline $\begin{array}{c}\text { Testing } \\
\text { Database 1 }\end{array}$ & 13.92 & 14.04 & 14.18 & 14.48 & 14.76 \\
\hline $\begin{array}{c}\text { Testing } \\
\text { Database 2 }\end{array}$ & 13.89 & 14.26 & 14.35 & 14.68 & 14.87 \\
\hline Average MAE & 13.91 & 14.15 & 14.27 & 14.58 & 14.82 \\
\hline
\end{tabular}

In Figure 17, we show some estimation results using our proposed method and OpenBR-age-estimation method. As shown in the figure, the estimated ages by our proposed method is closer to the ground-truth age than those by OpenBR method. The reason why our method outperforms the OpenBR-age-estimation system is that our proposed method assesses the blurring degree before performing the estimation step. Consequently, an appropriate age estimator is used for age estimation instead of using a global age estimator for all input images. 


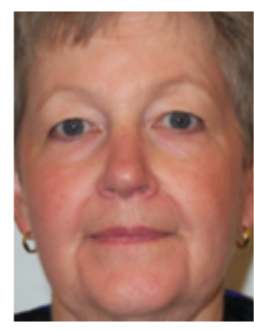

Sigma $=1$

Ground-truth: 64

Estimated Age by OpenBR: 55

Estimated Age by our Method: 55

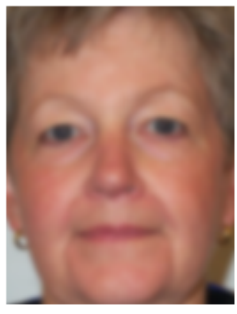

Sigma $=3$

Ground-truth: 64

Estimated Age by OpenBR: 52

Estimated Age by our Method: 59

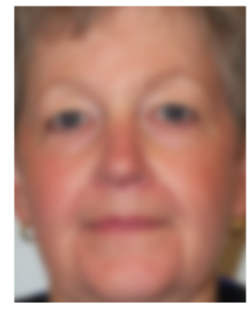

Sigma $=4$

Ground-truth: 64

Estimated Age by OpenBR: 49

Estimated Age by our Method: 66

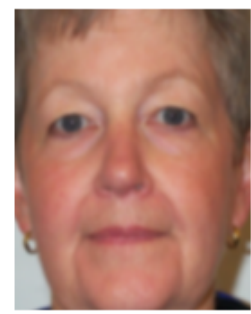

Sigma $=2$

Ground-truth: 64

Estimated Age by OpenBR: 53

Estimated Age by our Method: 63

(a)

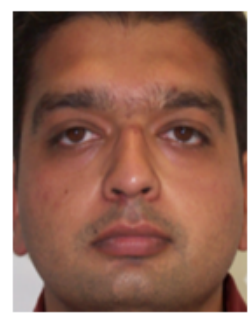

Sigma $=1$

Ground-truth: 27

Estimated Age by OpenBR: 33

Estimated Age by our Method: 22

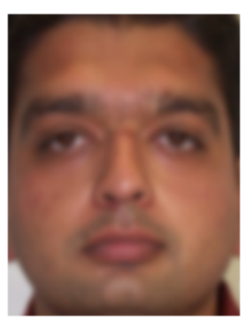

Sigma $=3$

Ground-truth: 27

Estimated Age by OpenBR: 36

Estimated Age by our Method: 26

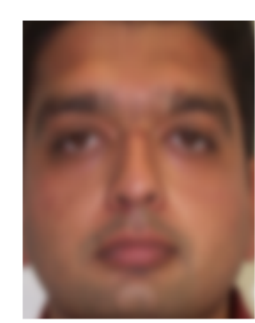

Sigma $=4$

Ground-truth: 27

Estimated Age by OpenBR: 36

Estimated Age by our Method: 28

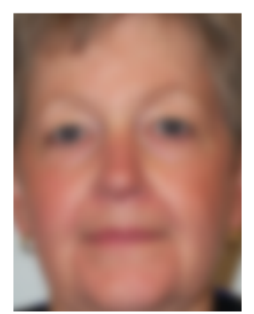

Sigma $=5$

Ground-truth: 64

Estimated Age by OpenBR: 48

Estimated Age by our Method: 66

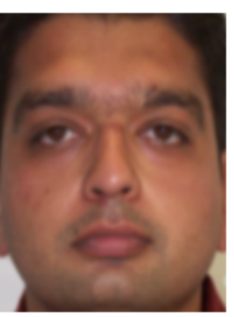

Sigma $=2$

Ground-truth: 27

Estimated Age by OpenBR: 37

Estimated Age by our Method: 27

(b)

Figure 17. Comparison of estimation results using OpenBR-age-estimation method and our proposed method using optical blurring images: (a) the estimation results of a female at the age of 64 with different values of optical blurring degree (sigma value of Gaussian kernel); and (b) the estimation results of a male at the age of 27 with different values of optical blurring degree. 


\section{Conclusions}

In this paper, we proposed a new human age estimation method with the advantage of considering the effects of image blurring. By assessing the FS of facial images, input face images are classified into one of five groups according to blurring degree. Using the classification results, the appropriate age estimator based on MLBP, Gabor filtering, and PCA with SVR trained with the data of the corresponding group, is activated for age estimation. The use of the PCA method allows our method to reduce the dimension of the age feature and enhance estimation performance. Through the experiment results, we confirmed the efficiency of our method over previous methods.

In future works, we plan to research various other factors that can affect the performance of age estimation systems, such as occlusion, low resolution, and motion blurring.

\section{Acknowledgments}

This study was supported by the SW R\&D program of MSIP/IITP (10047146, Real-time Crime Prediction and Prevention System based on Entropy-Filtering Predictive Analytics of Integrated Information such as Crime-Inducing Environment, Behavior Pattern, and Psychological Information).

\section{Author Contributions}

Dat Tien Nguyen and Kang Ryoung Park designed the overall system of age estimation. In addition, they wrote and revised the paper. So Ra Cho implemented the focus measurement method of optical blurring, and helped with the experiments.

\section{Conflict of Interests}

The authors declare no conflict of interest.

\section{References}

1. Fu, Y.; Guo, G.; Huang, T.S. Age synthesis and estimation via faces: A survey. IEEE Trans. Pattern Anal. Mach. Intell. 2010, 32, 1955-1976.

2. Guo, G. Human age estimation and sex classification. Video Anal. Bus. Intell. 2012, 409, 101-131.

3. Dantcheva, A.; Singh, A.; Elia, P.; Dugelay, J.-L. Search Pruning in Video Surveillance Systems: Efficiency-Reliability Tradeoff. In Proceedings of the IEEE International Conference on Computer Vision Workshops, Barcelona, Spain, 6-13 November 2011; pp. 1356-1363.

4. Jain, A.K.; Dass. S.C.; Nandakumar, K. Can Soft Biometric Traits Assist User Recognition? In Proceedings of the SPIE International Symposium on Defense and Security: Biometric Technology for Human Identification; SPIE: Bellingham, WA, USA, 25 August 2004; Volume 5404, pp. 561-572.

5. Dantcheval, A.; Dugelay, J.-L.; Elia, P. Soft Biometric System: Reliability and Asymptotic Bounds. In Proceedings of the 4th IEEE International Conference on Biometrics: Theory Applications and Systems, Washington, DC, USA, 17-19 September 2010; pp. 1-6. 
6. Batool, N.; Taheri, S.; Chellappa, R. Assessment of Facial Wrinkles as a Soft Biometrics. In Proceedings of the 10th IEEE International Conference and Workshops on Automatic Face and Gesture Recognition, Shanghai, China, 22-26 April 2013; pp. 1-7.

7. Han, H.; Jain, A.K. Age, Gender and Race Estimation from Unconstrained Face Images; MSU Technical Report MSU-CSE-14-5; MSU: State of Michigan, USA, 2014; pp. 1-9.

8. Ramanathan, N.; Chellappa, R.; Biswas, S. Computational methods for modeling facial aging: A survey. J. Vis. Lang. Comput. 2009, 20, 131-144.

9. Zhang, H.; Lao, S.; Kurata, T. Face Recognition with Consideration of Aging. In Proceedings of the IEEE International Conference on Automatic Face and Gesture Recognition and Workshops, Santa Barbara, CA, USA, 21-25 March 2011; p. 343.

10. Luu, K.; Ricanek, K., Jr.; Bui, T.D.; Suen, C.Y. Age Estimation Using Active Appearance Models and Support Vector Machine Regression. In Proceedings of the IEEE 3rd International Conference on Biometrics: Theory, Applications, and Systems, Washington, DC, USA, 28-30 September 2009; pp. 1-5.

11. Lanitis, A.; Taylor, C.J.; Cootes, T.F. Toward automatic simulation of aging effects on face images. IEEE Trans. Pattern Anal. Mach. Intell. 2002, 24, 442-455.

12. Lanitis, A.; Draganova C.; Christodoulou, C. Comparing different classifiers for automatic age estimation. IEEE Trans. Syst. Man Cybern. B 2004, 34, 621-628.

13. Choi, S.E.; Lee, Y.J.; Lee, S.J.; Park, K.R.; Kim, J. A Comparative Study of Local Feature Extraction for Age Estimation. In Proceedings of the 11th International Conference on Control, Automation, Robotics and Vision, Singapore, Singapore, 7-10 December 2010; pp. 1280-1284.

14. Nguyen, D.T.; Cho, S.R.; Shin, K.Y.; Bang, J.W.; Park, K.R. Comparative study of human age estimation with or without pre-classification of gender and facial expression. Sci. World J. 2014, 2014, 1-15.

15. Gunay, A.; Nabiyev, V.V. Automatic Age Classification with LBP. In Proceedings of the 23rd International Symposium on Computer and Information Sciences, Istanbul, Turkey, 27-29 October 2008; pp. 1-4.

16. Nguyen, D.T.; Cho, S.R.; Park, K.R. Human Age estimation based on multi-level local binary pattern and regression method. Lect. Notes Electron. Eng. 2014, 309, 433-438.

17. Li, C.; Liu, Q.; Liu, J.; Lu, H. Learning Ordinal Discriminative Features for Age Estimation. In Proceedings of the IEEE Conference on Computer Vision and Pattern Recognition, Providence, RI, USA, 16-21 June 2012; pp. 2570-2577.

18. Geng, X.; Zhou, Z.-H.; Smith-Miles, K. Automatic age estimation based on facial aging patterns. IEEE Trans. Pattern Anal. Mach. Intell. 2007, 29, 2234-2240.

19. Guo, G.; Mu, G.; Fu, Y.; Huang, T.S. Human Age Estimation Using Bio-inspired Features. In Proceedings of the IEEE Conference on Computer Vision and Pattern Recognition, Miami, FL, USA, 20-25 June 2009; pp. 112-119.

20. Viola, P.; Jones, M.J. Robust real-time face detection. Int. J. Comput. Vis. 2004, 57, 137-154.

21. OpenCV. Available online: http://opencv.org/ (accessed on 10 September 2015).

22. Wang, H.L.; Wang, J.-G.; Yau, W.-Y.; Chua, X.L.; Tan, Y.P. Effects of Facial Alignment for Age Estimation. In Proceedings of the 11th International Conference on Control, Automation, Robotics and Vision, Singapore, Singapore, 7-10 December 2010; pp. 644-647. 
23. Jang, J.; Park, K.R.; Kim, J.; Lee, Y. New focus assessment method for iris recognition system. Pattern Recognit. Lett. 2008, 29, 1759-1767.

24. Nayar, S.K.; Nakagawa, Y. Shape from focus. IEEE Trans. Pattern Anal. Mach. Intell. 1994, 16, 824-831.

25. Kautsky, J.; Flusser, J.; Zitova, B.; Simberova, S. A new wavelet-based measure of image focus. Pattern Recognit. Lett. 2002, 23, 1785-1794.

26. Kang, B.J.; Park, K.R. A robust eyelash detection based on iris focus measurement. Pattern Recognit. Lett. 2007, 28, 1630-1639.

27. Daugman, J. How iris recognition works. IEEE Trans. Circuits Syst. Video Technol. 2004, 14, 21-30.

28. Gesu, V.D.; Tabacchi, M.E.; Zavidovique, B. Symmetry as an intrinsically dynamic feature. Symmetry 2010, 2, 554-581.

29. Su, J.Y.; Cheng, S.C.; Huang, D.K. Unsupervised object modeling and segmentation with symmetry detection for human activity recognition. Symmetry 2015, 7, 427-449.

30. Choi, S.E.; Lee, Y.J.; Lee, S.J.; Park, K.R.; Kim, J. Age estimation using a hierarchical classifier based on global and local facial features. Pattern Recognit. 2011, 44, 1262-1281.

31. Nguyen, D.T.; Park, Y.H.; Lee, H.C.; Shin, K.Y.; Kang, B.J.; Park, K.R. Combining touched fingerprint and finger-vein of a finger, and its usability evaluation. Adv. Sci. Lett. 2012, 5, 85-95.

32. Fang, Y.; Wang, Z. Improving LBP Features for Gender Classification. In Proceedings of the International Conference on Wavelet Analysis and Pattern Recognition, Hong Kong, China, 30-31 August 2008; pp. 373-377.

33. Alomar, F.A.; Muhammad, G.; Aboalsamh, H.; Hussain, M.; Mirza, A.M.; Bebis, G. Gender Recognition from Faces Using Bandlet and Local Binary Patterns. In Proceedings of the 20th International Conference on Systems, Signals and Image Processing, Bucharest, Romania, 7-9 July 2013; pp. 59-62.

34. Shan, C.; Gong, S.; McOwan, P.W. Facial expression recognition based on local binary patterns: A comprehensive study. Image Vis. Comput. 2009, 27, 803-816.

35. Lee, W.O.; Kim, Y.G.; Hong, H.G.; Park, K.R. Face recognition system for set-top box-based intelligent TV. Sensors 2014, 14, 21726-21749.

36. Manjunath, B.S.; Ma, W.Y. Texture features for browsing and retrieval of image data. IEEE Trans. Pattern Anal. Mach. Intell. 1996, 18, 837-842.

37. Ahmadi, A.; Omatu, S.; Kosaka, T. A PCA Based Method for Improving the Reliability of Bank Note Classifier Machines. In Proceedings of the 3rd International Symposium on Image and Signal Processing and Analysis, Rome, Italy, 18-20 September 2003; pp. 494-499.

38. Murphy, K.P. Machine Learning: A Probabilistic Perspective; The MIT Press: Cambridge, MA, USA, 2012.

39. Song, F.; Guo, Z.; Mei, D. Feature Selection Using Principal Component Analysis. In Proceedings of the International Conference on System Science, Engineering Design and Manufacturing Information, Yichang, China, 12-14 November 2010; pp. 27-30.

40. Karim, T.F.; Rahman, M.L.; Lipu, M.S.H.; Sultana, F. Face Recognition Using PCA-Based Method. In Proceedings of the IEEE International Conference on Advanced Management Science, Chengdu, China, 9-11 July 2010; pp. 158-162. 
41. LibSVM-A Library for Support Vector Machines. Available online: http://www.csie.ntu.edu.tw/ cjlin/ libsvm/ (accessed on 10 September 2015).

42. Minear, M.; Park, D.C. A lifespan database of adult facial stimuli. Behav. Res. Methods 2004, 36, 630-633.

43. PAL Database. Available online: http://agingmind.utdallas.edu/facedb (accessed on 10 September 2015).

44. Gonzalez, R.C.; Woods, R.E. Digital Image Processing, 3rd ed.; Prentice Hall: Upper Saddle River, NJ, USA, 2010.

45. MORPH Database. Available online: https://ebill.uncw.edu/C20231_ustores/web/store_main.jsp? STOREID=4 (accessed on 10 September 2015).

46. OpenBR. Available online: http://openbiometrics.org/ (accessed on 10 September 2015).

(C) 2015 by the authors; licensee MDPI, Basel, Switzerland. This article is an open access article distributed under the terms and conditions of the Creative Commons Attribution license (http://creativecommons.org/licenses/by/4.0/). 